\title{
An Improved Firefly Algorithm-Based 2-D Image Thresholding for Brain Image Fusion
}

\author{
Srikanth M. V., Independent Researcher, India \\ V. V. K. D. V. Prasad, Jawaharlal Nehru Technological University, Kakinada, India \\ K. Satya Prasad, Jawaharlal Nehru Technological University, Kakinada, India
}

\begin{abstract}
In this article, an attempt is made to diagnose brain diseases like neoplastic, cerebrovascular, Alzheimer's, and sarcomas by the effective fusion of two images. The two images are fused in three steps. Step 1. Segmentation: The images are segmented on the basis of optimal thresholding, the thresholds are optimized with an improved firefly algorithm (pFA) by assuming Renyi entropy as an objective function. Earlier, image thresholding was performed with a 1-D histogram, but it has been recently observed that a 2-D histogram-based thresholding is better. Step 2: the segmented features are extracted with the scale invariant feature transform (SIFT) algorithm. The SIFT algorithm is good in extracting the features even after image rotation and scaling. Step 3: The fusion rules are made on the basis of an interval type-2 fuzzy set (IT2FL), where uncertainty effects are minimized unlike type-1. The novelty of the proposed work is tested on different benchmark image fusion data sets and has proven better in all measuring parameters.
\end{abstract}

\section{KEYWORDS}

Firefly Algorithm, Image Fusion, Image Thresholding, Interval Type-2 Fuzzy, Renyi Entropy, Scale Invariant Feature Transform

\section{INTRODUCTION}

Diagnosis of a disease is an important step to cure an illness. There are many diagnostic techniques and one of them is medical imaging. It is a technique to generate a visual representation of the desired part of the human body including tissues, bones and brain. Many multi-modal medical imaging techniques were introduced to achieve it. The main solution among them is Image fusion. Gathering required information from various images and fusing them together into a single image without any distortion and loss of information. Image fusion has many advantages, they include less ambiguity, improved reliability, image sharpening, feature enhancement, improved classification, diagnosing disease in early stages, easy to interpret, low in cost, reduces data transmission and fused image is true in color. Image fusion can be applied in medical diagnosis, satellite imaging, object detection and recognition, military, astronomy.

Many techniques and classifications have been originated in image fusion. The main techniques are spatial domain techniques (Ghassemian, 2016), transform domain techniques (Jin et al., 2017), 
contrast pyramid technique, ratio pyramid technique, morphological pyramid technique (Petrovic \& Xydeas, 2004), Laplacian pyramid technique, PCNN (Yang et al., 2009), pixel level image fusion technique (Naidu \& Rao, 2008), feature level image techniques (Nirmala \& Vaidehi, 2015, March), decision level image fusion techniques (Al-Tayyan et al., 2017). The spatial domain image fusion technique directly deals with the pixels of the input images. This technique can be analyzed in four ways namely, principal component analysis (PCA) (Wan et al., 2013), Intensity hue and saturation (IHS), Simple averaging, Simple maxima. Although they are very important techniques, they have certain limitations. Simple maxima produces highly focused images but it causes blurring (Xu, 2014). Local contrast can be affected by blurring. Simple averaging cannot give clear images of the object, but it is the simplest method for image fusion. Spectral degradation is produced due to principal component analysis technique. Intensity hue saturation is only suitable for color image fusion so it cannot be applied in medical imaging (Haddadpour et al., 2017).

In transform domain techniques, initially the transform of the function is analyzed and the resultant coefficients of transforms are fused. Then, the inverse transform is estimated to get fused images. In this technique, images are converted into multi-resolution or multi-scale representations before fusion. This technique can be analyzed using discrete wavelet transform (DWT), DT-CWT, Curvelet transform (Choi et al., 2015), additive wavelet transforms, non-sub sampled Contourlet transform (NSCT). Contourlet transform is used for catching complex contours, edges and textures (Yang et al., 2008). Few methods in the transform domain are far better than spatial domain methods in many aspects. Discrete wavelet transform cannot handle curved edges. Therefore, it fails in fusing images of the brain in a required manner. It has poor directionality, shift sensitivity, destruction of phase information, less spatial resolution. It has Pseudo-Gibbs effect due to the down-sampling process. Curvelet transform is specially designed for curved edges and capturing curvilinear properties. Therefore, it is beneficial in fusing the images of brain easily and clearly this method is very complex and it takes very high processing time. DT-CWT technique has minor drawbacks as compared to spatial domain analysis and transforms domain analysis. DT-CWT technique has high directionality, better edge representation, approximate shift invariant property and takes less time for processing (El-Hoseny et al., 2018).

The Pyramid techniques in image fusion are suitable only for specific type of images. Therefore, only one bandpass image is generated by pyramid level image fusion techniques and they often cause blocking effects. Apart from this, Contrast pyramid technique will lose much information while Morphological pyramid technique has bad edges. The pulse coupled neural network (PCNN) technique is very useful for image segmentation, image enhancement and pattern recognition. It has an important biological background. PCNN is used to produce images with high contrast, clarity and information. M-PCNN is very useful when several images are fused at a time and depending upon the actual requirements, number of channels can be easily changed. Still it has drawbacks, it is very difficult and complex to set the design constraints, and they have to be adjusted manually or they can be estimated through large amount of training, every parameter is important. The performance of PCCN image fusion is completely dependent on those parameters. To overcome this drawback intelligent optimization algorithms are used. They are GA-PCCN, PSO-PCCN, QPSO-PCCN, MDEPCNN and FOA-PCCN (Lang \& Hao, 2014). The pixel level can hold large amount of information and it also has high precision. The area may approach by which you can achieve pixel level image fusion. They are, intensity hue saturation, principal component analysis and multi-resolution analysis which is widely used.

To decide a technique which is rightly suitable for our application we should consider certain parameters like strength, orientation, offset of the image edges, ridges and other singularities. After deciding the technique, we move for global optimization techniques. To achieve our objective, global optimization is a task for finding the best set of admissible conditions. It is used to find the maxima and minima for continuous and differential functions. The techniques are firefly algorithm (FA), gray wolf optimization (GWO) (Karri \& Jena, 2016), Genetic Algorithms (GA) Symbiotic Organisms 
Search (SOS), particle swarm optimization (PSO) (Chiranjeevi \& Jena, 2017), Honeybee mate optimization (HBMO) (Chiranjeevi \& Jena, 2018). Among them, improved firefly algorithm (pFA) has more advantageous like simple mathematics, ease of implementation, high processing time and less convergence speed. Soon after completion of image fusion techniques we have to face two main challenges image contrast and visual quality.

\section{PROPOSED IMAGE FUSION}

In this paper, a region-based feature level image fusion is proposed, the regions of both the images which are to be fused are obtained by perfect image segmentation. The images are segmented for extraction of similar regions or areas in both the images by optimal thresholds. The optimal thresholds are obtained using suitable optimization techniques by assuming Renyi entropy as an objective function. These thresholds are optimized based on the 2-D histogram of image to be fused. The task of optimization technique is to maximize the objective function which leads to better threshold, henceforth improved segmentation of input images. The thresholds are optimized with the improved firefly algorithm and compared the results with some other state of art optimization techniques. After successful segmentation of both the images, the features of the input images are extracted with a suitable feature extraction technique. In general, the features of an image are texture, edge and contour. In this paper, image features are extracted with Scale Invariant Feature Transform (SIFT) algorithm, it was invented by (Lowe, 1999) and the working principal of the algorithm starts with random extraction of objects from reference image and new object from other image is recognized on the basis of minimum Euclidean distance between the reference and object which is to be detected. The detailed description about the algorithm is found in reference number (He et al., 2018). The extracted features of both the images are fused based on type - 2 fuzzy fusion rules. Theory of fuzzy set was created in 1965 by Zadeh (Zadeh, 1965). However, it is found that type-1 fuzzy set is not adaptive and flexible, therefore, similarly proposed a type -2 fuzzy set, and in which membership function is uncertain because of uncertainty in membership relationship. So, it is adaptable for both certain and uncertain problems. Figure 1 shows the block diagram of proposed algorithm.

Image thresholding: Image thresholding is one of the image clustering technique. It is used in many applications like image processing, data mining, data clustering etc., because of its simple and flexible nature. Image thresholding may be bi-level or multi-level, in first case two thresholds are used for segmentation of the given image into three regions. Whereas, in second case, number of thresholds is fixed by the user depending on his requirement. If number of thresholds are ' $c$ ' then the number of clusters or segments are ' $c+1$ '. The segmented result depends on perfect placement of thresholds which segment the input image. Placing these thresholds is difficult task for different images. So, in this, these thresholds are optimized with improved firefly algorithm (pFA) by considering Renyi entropy as objective function.

\section{MATERIALS AND METHODS}

In this section a brief review of Renyi entropy, improved firefly algorithm (pFA), Scale-Invariant Feature Transform, Type-2 Fuzzy Set and Fusion Rules are discussed.

\section{Concept of Renyi Entropy}

Let's assume an ' $n$ ' array finite discrete probability distributions (pdf) such as $\left(\mathrm{F}_{1}, \mathrm{~F}_{2}, \mathrm{~F}_{3}, \ldots \ldots \mathrm{F}_{\mathrm{n}}\right) \varepsilon$ $\Delta_{\mathrm{n}}$ where $\Delta_{\mathrm{n}}=\left\{\left(\mathrm{F}_{1}, \mathrm{~F}_{2}, \mathrm{~F}_{3}, \ldots \ldots \mathrm{F}_{\mathrm{n}}\right), \mathrm{F}_{\mathrm{i}} \geq 0, \mathrm{i}=1,2,3 \ldots \ldots \mathrm{n}, \mathrm{n} \geq 2, \sum_{i=1}^{n} F_{i}=1\right\}$ with random variables 
Figure 1. Block diagram of proposed technique

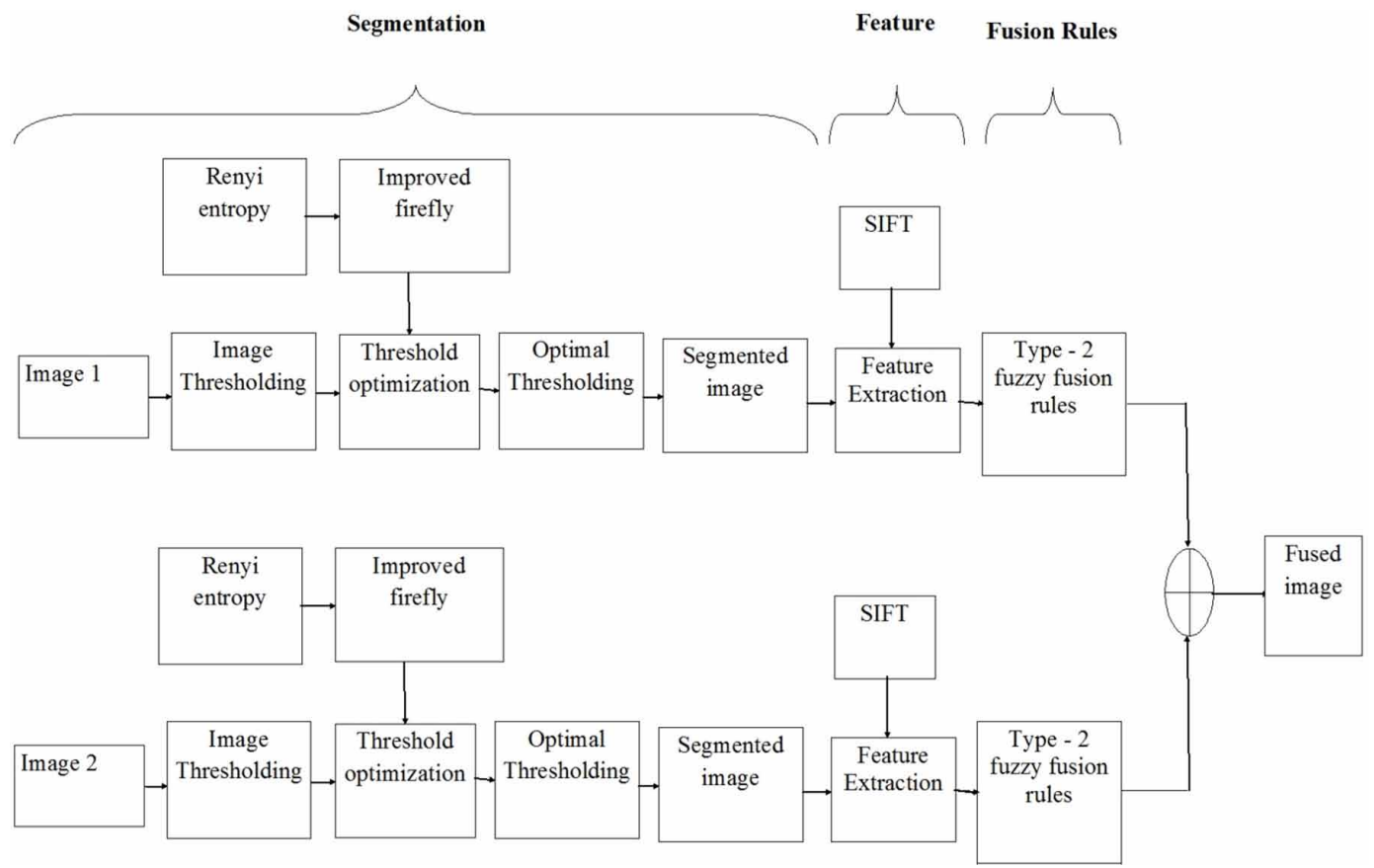

$\left(\mathrm{X}_{1}, \mathrm{X}_{2}, \mathrm{X}_{3}, \ldots \ldots \mathrm{X}_{\mathrm{n}}\right)$ then Renyi entropy for independent and additive random events is given as (Rényi, 1960)

$$
H_{\alpha}=\frac{1}{1-\alpha} \log _{2}\left(\sum_{i}^{n} F_{i}^{\alpha}\right)
$$

where ' $\alpha$ ' is greater than zero and it is called as entropy order. When ' $\alpha$ ' tends to one then Renyi entropy becomes Shannon entropy. In general image is clustered in to two clusters, one carries object information (cluster $\mathrm{C}_{1}$ ) and another carries background (cluster $\mathrm{C}_{2}$ ) then Renyi entropy is:

$$
\begin{aligned}
& H_{\alpha}\left[C_{1}\right]=\frac{1}{1-\alpha}\left[\log _{2}\left(\sum_{i=0}^{t}\left(\frac{F(i)}{F\left(C_{1}\right)}\right)^{\alpha}\right)\right] \\
& H_{\alpha}\left[C_{2}\right]=\frac{1}{1-\alpha}\left[\log _{2}\left(\sum_{i=t}^{L-1}\left(\frac{F(i)}{F\left(C_{2}\right)}\right)^{\alpha}\right)\right]
\end{aligned}
$$


where $F\left(C_{1}\right)=\sum_{i=0}^{t} F(i), F\left(C_{2}\right)=\sum_{i=t+1}^{L-1} F(i)$, Here $F_{i}$ is the normalized one dimensional histogram of the image and ' $L$ ' is highest intensity level of gray scale image. The overall renyi entropy for a given image with one threshold ' $\mathrm{t}$ ' is given as:

$$
\varphi_{\alpha}(t)=\operatorname{argmax}\left(\left[H_{\alpha}\left[C_{1}\right]+H_{\alpha}\left[C_{2}\right]\right]\right)
$$

\section{Multi-Level Thresholding}

Let image is divided into ' $N$ ' number of clusters $\mathrm{C}=\left(\mathrm{C}_{1}, \mathrm{C}_{2}, \mathrm{C}_{3}, \ldots \ldots \mathrm{C}_{\mathrm{N}}\right)$ with $\mathrm{N}$ number of threshold values $t=\left(t_{1}, t_{2}, t_{3}, \ldots . . t_{N}\right)$ then Renyi entropy for each individual cluster is defined as:

$$
H_{\alpha}\left[C_{1}\right]=\frac{1}{1-\alpha}\left[\log _{2}\left(\sum_{i=0}^{t_{1}}\left(\frac{F(i)}{F\left(C_{1}\right)}\right)^{\alpha}\right)\right]
$$

$$
H_{\alpha}\left[C_{2}\right]=\frac{1}{1-\alpha}\left[\log _{2}\left(\sum_{i=t_{1}+1}^{t_{2}}\left(\frac{F(i)}{F\left(C_{2}\right)}\right)^{\alpha}\right)\right]
$$

$$
H_{\alpha}\left[C_{N}\right]=\frac{1}{1-\alpha}\left[\log _{2}\left(\sum_{i=t_{N}-1}^{L-1}\left(\frac{F(i)}{F\left(C_{N}\right)}\right)^{\alpha}\right)\right]
$$

where $F\left(C_{1}\right)=\sum_{i=0}^{t_{1}} F(i), F\left(C_{2}\right)=\sum_{i=t_{1}+1}^{t_{2}} F(i), F\left(C_{N}\right)=\sum_{i=t_{N}-1}^{L-1} F(i)$, the overall Renyi entropy or objection function for a given image for $\mathrm{N}$ thresholds is given as:

$$
\varphi_{\alpha}(t)=\operatorname{argmax}\left(\left[H_{\alpha}\left[C_{1}\right]+H_{\alpha}\left[C_{2}\right]+\ldots H_{\alpha}\left[C_{N}\right]\right]\right)
$$

For simplifying the calculations, two dummy thresholds are introduced $t_{0}$ and $t_{\mathrm{N}}=\mathrm{L}-1$ which satisfy the condition $\mathrm{t}_{0}<\mathrm{t}_{1} \ldots<\mathrm{t}_{\mathrm{N}-1}<\mathrm{t}_{\mathrm{N}}$. The optimal thresholds are obtained by maximizing the above equation with any soft computing technique.

\section{Two-Dimensional Renyi Entropy}

Let $\mathrm{I}(m, n)$ is an image intensity at spatial location $(m, n)$. In digital image $[I(\mathrm{~m}, \mathrm{n}) \mid m \varepsilon\{1,2,3, \ldots$, $M\}, \mathrm{n} \varepsilon\{1,2,3, \ldots, N\}$, where ' $M$ ' and ' $N$ ' are size of the image and its $1 \mathrm{D}$-histogram $\mathrm{h}(\mathrm{x})$ for $x \varepsilon$ $\{1,2,3, \ldots, L-1\}$, where ' $L$ ' is 256 for gray scale image. Let denote elements in histogram $\{1,2,3 \ldots$ 
255 \} as G. In literature, optimal thresholds selection is based on 1D-histogram and are obtained by optimizing the objective function/entropy.

The 2-D histogram of an image is obtained by defining a local average of pixel, $I(x, y)$, as the average intensity of its nine neighbors denoted as $g(x, y)$ (Sarkar \& Das, 2013):

$g(x, y)=\frac{1}{9} \sum_{i=-1}^{1} \sum_{j=-1}^{1} f(x+i, y+j)$

For example let us take an image of size $4 * 4$ as shown in Figure 2 (a) and its average intensity $g(x, y)$ is calculated by padding required number of zeros at edges as shown in Figure 2 (b). First table

Figure 2. Example for 2-D histogram calculation

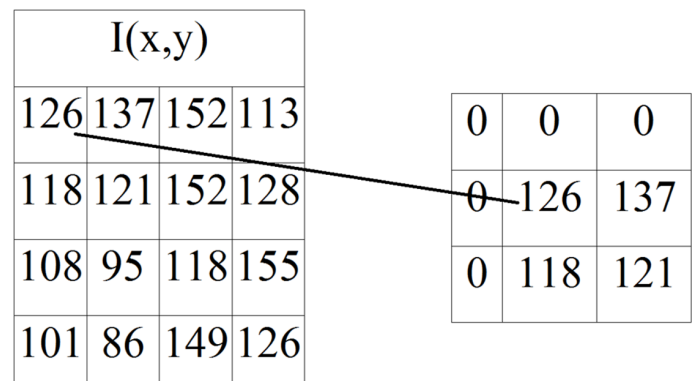

\begin{tabular}{|c|c|c|c|}
\hline \multicolumn{4}{|c|}{$g(x, y)$} \\
\hline 56 & 90 & 89 & 61 \\
\hline 78 & 125 & 130 & 91 \\
\hline 70 & 116 & 126 & 92 \\
\hline 43 & 73 & 81 & 61 \\
\hline
\end{tabular}

in Figure 2 is image and first element i.e 126, $g(x, y)$ is calculated by padding zero's at edges as in Figure 2 and last tables shows $g(x, y)$ for entire image $I(x, y)$. 2-D histogram of Lena image at marked area is shown in Figure 3, where diagonal quadrants carry much information.

The 2-D histogram of tested images as shown in Figure 3 and are divided into four clusters by a single threshold $(t, s)$. Where $t$ is threshold for original image intensity $I(x, y)$ and $s$ is threshold for average intensity image $g(x, y)$. The divided cluster area is not same. The diagonal quadrant $1^{\text {st }}$ represents object and $3^{\text {rd }}$ represent background and $2^{\text {nd }}$ and $4^{\text {th }}$ quadrants are neglected because does

Figure 3. Lena image and 2-D histogram

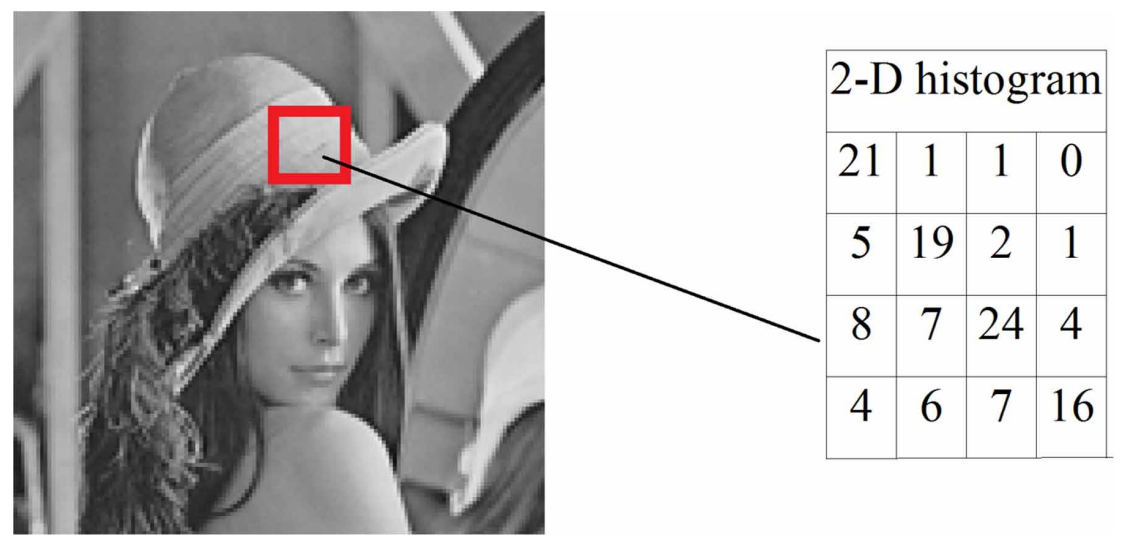


not carry any information (pair occurrence is less) as show in Figure 3. The Renyi entropy for object and background is given as:

$H_{\text {object }}^{\alpha}[t, s]=\frac{1}{1-\alpha}\left[\log _{2} \sum_{i=0}^{t}\left(\sum_{j=0}^{s}\left(\frac{F(i, j)}{F_{D 1}(t, s)}\right)^{\alpha}\right)\right]$

$H_{\text {background }}^{\alpha}[t, s]=\frac{1}{1-\alpha}\left[\log _{2} \sum_{i=t+1}^{L-1}\left(\sum_{j=s+1}^{L-1}\left(\frac{F(i, j)}{F_{D 2}(t, s)}\right)^{\alpha}\right)\right]$

where:

$F_{D 1}(t, s)=1-\sum_{i=0}^{t} \sum_{j=0}^{s} F(i, j)$ and $F_{D 2}(t, s)=1-\sum_{i=t+1}^{L-1} \sum_{j=s+1}^{L-1} F(i, j)$

The final objective function which is to maximized for better threshold $(t, s)$ selection is:

$\varphi_{\alpha}(t)=\operatorname{argmax}\left(\left[H_{\text {object }}^{\alpha}[t, s]+H_{\text {background }}^{\alpha}[t, s]\right]\right)$

\section{Proposed Renyi 2D-Hisotgram Based Multi-Level Thresholding}

Multilevel thresholding with 1-D histogram deliver inferior results because of incorrect selection of thresholds, so recent study proved that thresholding with 2-D histogram deliver superior results especially in multilevel thresholding. Multilevel thresholding gained lots of popularity over bi- level thresholding because, it clusters the image into several useful clusters, helps for accurate analysis and interpretation of the image. In this paper, we proposed a 2-D Renyi entropy based multilevel thresholding for image segmentation by incorporating the advantage of 2-D histogram. If the 2-D histogram of an image is cluster into 9 clusters with two thresholds $\left(t_{1}, t_{2}\right)$ and $\left(s_{1}, s_{2}\right)$ as shown in Figure 4 (a). Then the diagonal quadrants $1^{\text {st }}, 5^{\text {th }}$ and $9^{\text {th }}$ represents objects(s), intermediate regions and background respectively as illustrated in Figure 4 (a) and reset of the regions are noise and edges and are ignored. The Renyi entropy of diagonal quadrants are given as:

$$
\begin{aligned}
& H_{\text {object }}^{\alpha}[t, s]=\frac{1}{1-\alpha}\left[\log _{2} \sum_{i=0}^{t_{1}}\left(\sum_{j=0}^{s_{1}}\left(\frac{F(i, j)}{F_{D 1}(t, s)}\right)^{\alpha}\right)\right] \\
& H_{\text {intermediate }}^{\alpha}[t, s]=\frac{1}{1-\alpha}\left[\log _{2} \sum_{t_{1}+1}^{t_{2}}\left(\sum_{s_{1}+1}^{s_{2}}\left(\frac{F(i, j)}{F_{D 2}(t, s)}\right)^{\alpha}\right)\right]
\end{aligned}
$$


Figure 4. 2-D histogram for a) 3- level b) 4- level

\begin{tabular}{|c|c|c|c|c|}
\hline \multicolumn{2}{|c|}{$(0,0)$} & \multicolumn{3}{|c|}{$\mathrm{t}_{2} \quad(0, \mathrm{~L}-1)$} \\
\hline \multirow[b]{2}{*}{$\mathrm{S}_{1}$} & & 2 & 3 & \\
\hline & 4 & 5 & 6 & \\
\hline $\mathbf{S}_{2}$ & 7 & 8 & 9 & \\
\hline \multicolumn{2}{|c|}{$(\mathrm{L}-1,0)$} & & & \\
\hline
\end{tabular}

(a)

\begin{tabular}{|c|c|c|c|c|c|}
\hline \multicolumn{6}{|c|}{$\begin{array}{lllll}(0,0) & \mathrm{t}_{1} & \mathrm{t}_{2} & \mathrm{t}_{3} & (0, \mathrm{~L}-1)\end{array}$} \\
\hline \multirow{2}{*}{$\mathrm{s}_{1}$} & 1 & 2 & 3 & 4 & \\
\hline & 5 & 6 & 7 & 8 & \\
\hline \multirow{2}{*}{$\mathrm{s}_{3}$} & 9 & 10 & 11 & 12 & \\
\hline & 13 & 14 & 15 & 16 & \\
\hline \multicolumn{2}{|c|}{$(\mathrm{L}-1,0)$} & & & $\mathrm{L}-1$, & $-1)$ \\
\hline
\end{tabular}

(b)

$H_{\text {backgroud }}^{\alpha}[t, s]=\frac{1}{1-\alpha}\left[\log _{2} \sum_{t_{2}+1}^{L-1}\left(\sum_{s_{2}+1}^{L-1}\left(\frac{F(i, j)}{F_{D 3}(t, s)}\right)^{\alpha}\right)\right]$

where:

$$
\begin{aligned}
& F_{D 1}(t, s)=1-\sum_{i=0}^{t_{1}} \sum_{j=0}^{s_{1}} F(i, j), F_{D 2}(t, s)=1-\sum_{i=t_{1+1}}^{t_{2}} \sum_{j=s_{1}+1}^{s_{2}} F(i, j) \text { and } \\
& F_{D 2}(t, s)=1-\sum_{i=t_{2+1}}^{L-1} \sum_{j=s_{2}+1}^{L-1} F(i, j)
\end{aligned}
$$

The final objective function which is to maximized for better threshold $(t, s)$ selection is:

$\varphi_{\alpha}(t)=\operatorname{argmax}\left(\left[H_{\text {object }}^{\alpha}[t, s]+H_{\text {intermediate }}^{\alpha}[t, s]+H_{\text {background }}^{\alpha}[t, s]\right]\right)$

Above equation can be extended for ' $N$ ' threshold values as given below:

$\varphi_{\alpha}(t)=\operatorname{argmax}\left(H_{1}^{\alpha}[t, s]+H_{2}^{\alpha}[t, s]+H_{3}^{\alpha}[t, s] \ldots H_{N+1}^{\alpha}[t, s]\right)$

where:

$$
H_{k}^{\alpha}[t, s]=\frac{1}{1-\alpha}\left[\log _{2} \sum_{i=t_{k-1}+1}^{t_{k}}\left(\sum_{j=s_{k-1}+1}^{s_{k}}\left(\frac{F(i, j)}{F_{D k}(t, s)}\right)^{\alpha}\right]\right.
$$


For simplifying the calculations, two dummy thresholds are introduced $\mathrm{t}_{0}$ and $\mathrm{t}_{\mathrm{N}}+1=\mathrm{L}-1$ which satisfy the condition $\mathrm{t}_{0}<\mathrm{t}_{1} \ldots<\mathrm{t}_{\mathrm{N}-1}<\mathrm{t}_{\mathrm{N}}+1$. Similarly, two dummy variable $\mathrm{s}_{0}$ and $\mathrm{s}_{\mathrm{N}}+1=\mathrm{L}-1$ which satisfy the condition $\mathrm{s}_{0}<\mathrm{s}_{1} \ldots<\mathrm{s}_{\mathrm{N}-1}<\mathrm{s}_{\mathrm{N}}+1$. The 2-D histogram of four standard images are shown in Figure 5 and form these it is observed that most of the information/energy is concentrated on

Figure 5. Input images and corresponding 2-D histogram
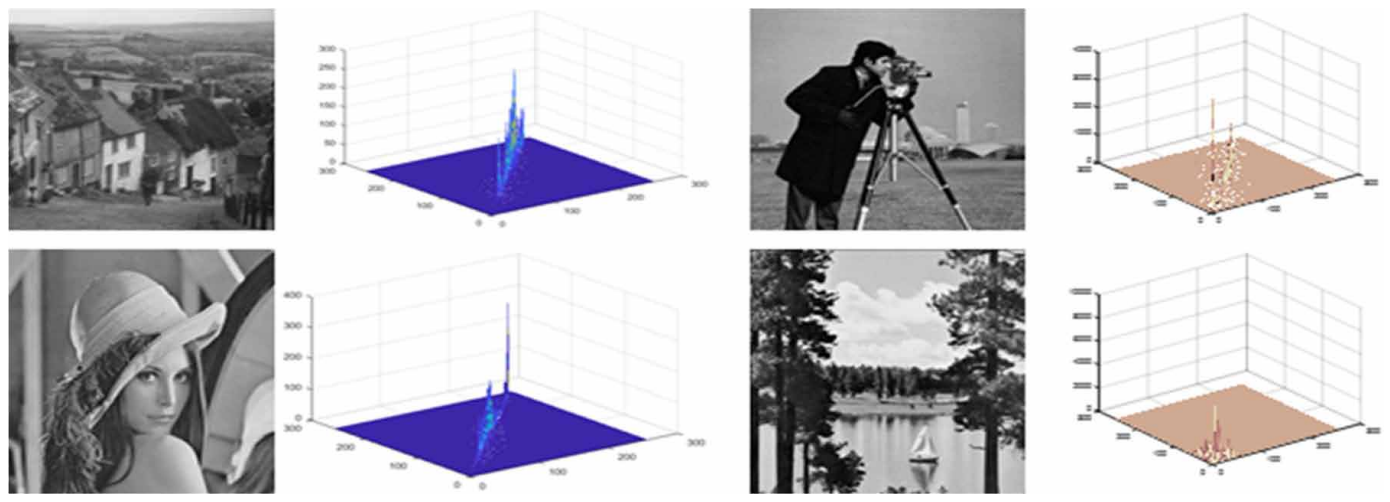

diagonal quadrants. Multilevel thresholding is a time-consuming process and is proportional to the number of thresholds 'N'. So, soft computing techniques play a significant role in this contest by assuming Equation (17) as an objection function, which leads to reduction in the computational time.

\section{FIREFLY ALGORITHM}

In FA, it mainly imitates the flashing behavior among fireflies. Each firefly is considered as a potential solution in search space, and the move behavior among firefly's stands for solution's upgrading to find a better solution. Moreover, in order to guarantee the algorithm on the right road, it is idealized by 3 hypotheses which are depicted as follows:

1. Fireflies are asexual; namely, they can be attracted with each other regardless of gender;

2. The size of the attraction is proportional to the brightness of the flashing. For two random fireflies $x_{i}$ and $x_{j}$, if $x_{i}$ gloomier than $x_{j}$, then $x_{i}$ is attracted by $x_{j}$. If $x i$ is the best one among all fireflies, it will move randomly in search space;

3. The brightness of fireflies is relative with the value of objective function.

\section{Basic Parameters}

In FA, both brightness $(I)$ and attractiveness $(\beta)$ play an important role. In general, for maximum problem, the brightness of a firefly is positively relative with the value of objective function, and for minimum problem, they are negatively relative with each other. Prior to defining the attractiveness (namely $\beta$ ), firstly, the Euclidean distance of $x_{i}$ and $x_{j}$ should be calculated as follows:

$r_{i j}=\sqrt{\sum_{i=1}^{D}\left(x_{i t}-x_{i t}\right)^{2}}$ 
where $\mathrm{D}$ is the dimension of the problem. Then, attractiveness is described as follows:

$\beta=\beta_{0} \times e^{-\gamma \times r_{i j}^{2}}$

\section{The Movement of the Firefly}

Supposed that $x_{i}$ is attracted by $x_{j}$, and then, $x_{i}$ will move as following:

$x_{i}^{\mathrm{t}+1}=\mathrm{x}_{\mathrm{i}}^{\mathrm{t}}+\beta \times\left(x_{j}^{t}-x_{i}^{t}\right)+\alpha \times($ rand -0.5$)$

where $x_{I}^{t}$ represents the position of $x i$ at the $t$-th iteration. $r a n d \in[0,1], \alpha$ is the random step, and $\alpha \in[0,1]$.

\section{The Detailed Step of the FA}

Step 1: Initialize population $N P$, and set parameters, such as $\beta_{0}$ and $\alpha$.

Step 2: Calculate $r_{i j}$ and $\beta$ with Formula (19) and (20), respectively.

Step 3: Upgrade solution with Formula (21).

Step 4: Generate a better solution by comparing the previous solution with the current solution.

Step 5: For the new solution, calculate its value of objective function.

Step 6: Judge whether the algorithm satisfies the terminate condition (up to the max number of iterations (ItMax)).

If satisfied, the algorithm end, meanwhile, output optimal solution; if not, return to Step 4.

\section{The Improved FA: pFA}

In FA, each firefly can be attracted by too many other fireflies, which caused severe oscillation. Thus, in order to alleviate this disadvantage, we proposed an improved algorithm abbreviated as pFA. In pFA, the attraction among fireflies is reduced greatly, and it can also lower computational time complexity and increase the accuracy of solution. The detailed description is as follows.

In $\mathrm{pFA}$, firstly, we calculate the value of fitness $\left(f_{i t}\right)$ of each firefly $\mathrm{x}_{\mathrm{i}}$ Formula (6):

$$
\begin{aligned}
& \text { fit }=\left\{\frac{1}{1+f\left(x_{i}\right)}, \text { if } f\left(x_{i}\right), f\left(x_{i}\right), \geq 0\right. \\
& 1+\left|f\left(x_{i}\right)\right|, \text { else }
\end{aligned}
$$

where $f(x)$ is objective function. And then, those values of fitness are bigger than $x_{i}$ 's (namely $i-t h$ firefly's) are put into a set $K$. Finally, we utilize the roulette wheel selection to choose its neighbor, the formula is shown as the following:

$$
\mathrm{p}_{\mathrm{fit}}=\frac{f_{i t}\left(x_{k}\right)}{\sum_{x_{t \in k}} f i t\left(x_{t}\right)}
$$


For $\mathrm{X}_{\mathrm{i}}$ randomly select a firefly $x_{k}$ as its neighbor with $p_{f i t}$ in set $K$, not in all fireflies. By this way, the worse fireflies are sifted out, and the remainders are better than $x_{i}$. Furthermore, from (21), we can know, the better the firefly is, the more chances to be chosen it has. Thus, no matter which one is selected, the chosen one will always give a good direction to lead $x_{i}$ rightly. Evidently, this strategy can not only speed up the convergence and guarantee the diversity of population, but also reduce selection pressure for fireflies. However, there exists such case that no firefly is better than $x_{i}$, that is to say $x_{i}$ is the best one in the whole population. Thus, it falls into local optimum easily. To deal with this case, the opposite learning skill is adopted by utilizing the information of $x_{i}$. Based on the above discussion, we can rewrite Formula (3) as follows:

$$
X_{i}^{t}= \begin{cases}x_{i}^{t}+e^{-\gamma r_{i j}^{2}}\left(x_{k}^{t}-x_{i}^{t}\right)+\alpha(\text { rand }-0.5), & \text { if } k=\Phi \\ l+u-x_{i}^{t}, & \text { else }\end{cases}
$$

As far as the step $\alpha$, this paper employees the following formula to update:

$$
X_{i}^{t+1}= \begin{cases}\alpha=0.25, & \text { if } t=1 \\ \alpha_{t}=\alpha_{0} \alpha^{t-1}, & \text { else }\end{cases}
$$

Here $\alpha_{0}=0.7$. And the $\alpha_{0}$ 's scope should be between 0 and 1 .

The detailed description of pFA is given in Algorithm 1.

Algorithm 1: Pseudo-code of pFA

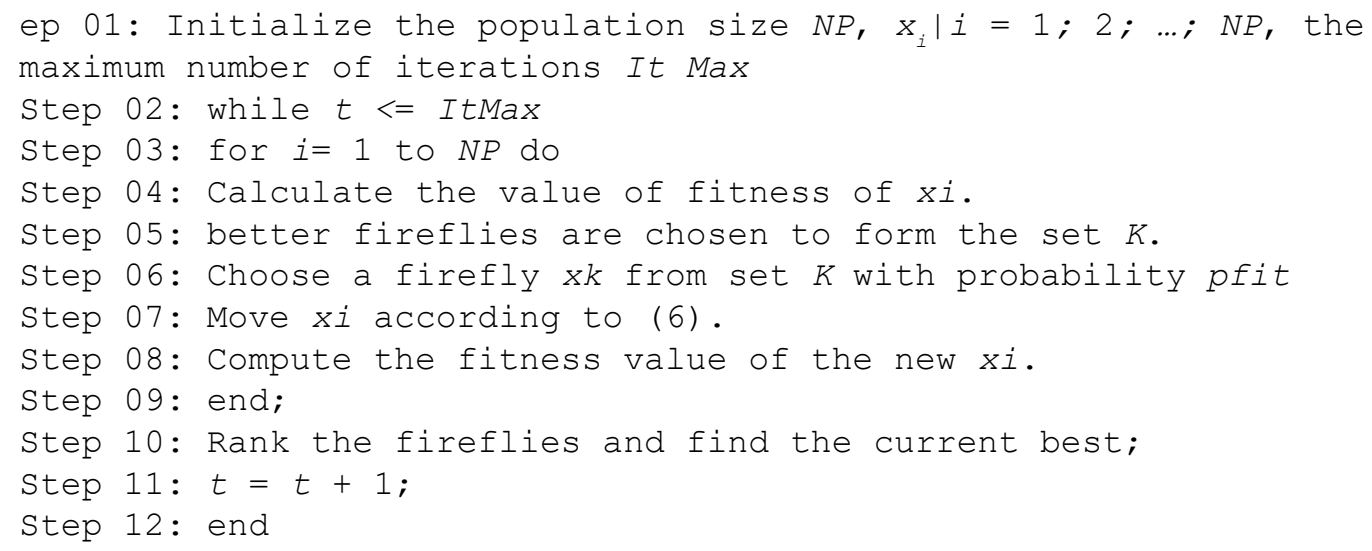

\section{Time Complexity}

For the optimization problem $(f)$, suppose that $O(f)$ is computational time complexity of evaluating its function value. Thus, the computational time complexity of FA is $O(\operatorname{ItMax} * N P 2 * f)$, while the computational time complexity of firefly algorithm with random attraction (RaFA) (Wang et al., 2016) and firefly algorithm with neighborhood attraction (NaFA) (Wang et al., 2017) are $O$ (ItMax $* N P * f)$ and $O($ ItMax $* k * N P * f)$, respectively, $k$ is the number of neighbor in NaFA. In this paper, for $\mathrm{pFA}$, its computational time complexity is $O(\operatorname{ItMax} * N P * f)$. Apparently, when it comes to computational time complexity, NaFA is equal to pFA, however, considering the accuracy and convergence speed of the algorithm, pFA is still better than NaFA. 


\section{Experiments on Benchmark Functions}

In this section, the performance of pFA is proved on 21 common benchmark functions derived from CEC 2005 and compared with FA, RaFA, and NaFA. And these functions derived from CEC 2005, where $f_{1}-f_{9}$ are unimodal functions, $f_{10}$ is noise function, $f_{11}-f_{18}$ are multimodal functions, $f_{19}$ and $f$ ${ }_{20}$ are orthogonal functions and $\mathrm{f} 21$ is shifted sphere function. The specific descriptions of these functions are shown in Table 1.

\section{Parameter Setting}

To make it fair, for all algorithms, the population size NP is set to 40, and the maximum iterations (ItMax) is set to 2500. Both the initial $\beta 0$ and $\gamma$ are set to 1 , and except for pFA, $\alpha=0: 25$. The $\alpha$ in pFA is discussed in Experiment 1: Moreover, for NaFA, $\mathrm{K}$ is set to 3(the number of neighbors). Furthermore, to deeply analyze the performance of pFA statistically, all algorithms run 30 times on 30, 50 and 100 dimension, respectively, and we take minimum, mean and deviation as the evaluation criterion to analyze the statistical results.

\section{Experiment 1: Determine the Value of the $\alpha_{0}$ and $\alpha_{1}$ in Formula (9)}

The better values of $\alpha_{0}$ and $\alpha_{1}$ in Formula (9) play a vital role in pFA. In the early stages of the iteration, the larger value of $\alpha$ can enhance the global search capability of the algorithm. As the iteration progresses, the smaller value of $\alpha$ can give the algorithm a strong local search ability. Thus, the $\alpha$ should be regressive with iterating, Now, we set $\alpha_{0}$ to $0.1,0.3,0.5,0.7,0.9$ and $\alpha_{1}$ is set to $0.15,0.35,0.55,0.75$, and 0.95 . And compare them on 21 benchmark functions with 30 dimension. Through experiment, we determine which value among them is the best for pFA. The results are shown in Table 2 and Table 3.

According to the data given in Table 3, for most functions, the best value of $\alpha_{0}$ is chosen in $[0: 5 ; 0: 7]$.

\section{Experiment 2: Comparison With Other FAs on Min, Worst, Mean and Standard Deviation}

In this part, to prove the superiority of pFA, we compare it with FA, RaFA and NaFA on 21 benchmark functions, which described in Table 1 .

When the dimension of the problem is 30, the computational results of FA, RaFA and NaFA and pFA are given in Table 4. Observing the table, we can know, for the mean, minimum and standard deviation, $\mathrm{pFA}$ is better than others on $f_{2}-f_{21}$. Among them, for $f_{5}-f_{9} ; f_{11} ; f_{13} ; f_{17}$ and $f_{20}$, pFA can find the minimum value of 0 , and even the mean and standard deviation are 0 , which demonstrates its high accuracy and better robustness; for $f_{3}, f_{4}, f_{10}, f f_{16}$ and $f_{19}$, the minimum value found by pFA is close to 0 . Moreover, both of FA and pFA can find the minimum value of 0 on $f_{1}$ and $f_{6}$, while For f18, $\alpha_{0}=0: 9$ is best, and for $f_{19}, f_{21}, \alpha_{0}=0: 7$ is better than others. In Table 4, for $f_{3}$ and $f_{14}, \alpha_{0}=$ $0: 55=0: 75$ is best, except them, its better that the value of $\alpha_{1}$ is chosen in [0:15;0.35] based on overall consideration. Thus, it is recommended that this combination, $\alpha_{1}=0: 7 ; \alpha_{1}=0: 25$, should be selected. RaFA and NaFA fail to find it. Finally, for $f_{14,} f_{15} f_{18,}$ and $f_{21}$, pFA is nearly equal to others. The computational results on 50 and 100 dimension are given in Table 5 and Table 6. Comparing with the results in Table 6, we can see, with dimension increasing, the mean, minimum and standard deviation of FA, RaFA and NaFA are all getting worse, while the results of pFA is almost invariable. It shows that $\mathrm{pFA}$ is still effective in solving high dimensional problems.

In order to further analyze the performance of $\mathrm{pFA}$, we give some convergent curve graphs of $f_{3 \text {, }}$ $f_{7}, f_{11}, f_{15}, f_{19}$, and $F_{21}$ on 30,50 and 100 dimension, respectively. The results are shown in Figure 6, Figure 7 and Figure 8. In Figure 6, for $f_{3}$, pFA almost converge to 0 when the iteration ends, and all of FA, RaFA and NaFA present slow increasing trend throughout the iteration process. For $f_{7}, f_{11}$ and 


\begin{tabular}{|c|c|c|}
\hline Functions & Range & $\begin{array}{c}\text { Optimal } \\
\text { Value }\end{array}$ \\
\hline $\mathrm{f} 1=\sum_{i=1}^{D} x_{i}^{2}$ & {$[-100.100]$} & 0 \\
\hline $\mathrm{f} 2=\sum_{i=1}^{D}\left|x_{i}\right|+\prod_{i=1}^{D}\left|x_{i}\right|$ & {$[-10.10]$} & 0 \\
\hline $\mathrm{f} 3=\sum_{i=1}^{D}\left(\sum_{j=1}^{i} x_{j}\right)$ & {$[-100.100]$} & 0 \\
\hline $\mathrm{f} 4=\max _{i}\left\{\left|x_{i}\right|, 1 \leq i \leq D\right\}$ & {$[-100.100]$} & 0 \\
\hline $\mathrm{f} 5=\sum_{i=1}^{D} i x_{i}^{2}$ & {$[-100.100]$} & 0 \\
\hline $\mathrm{f} 6=\sum_{i=1}^{D} i x_{i}^{4}$ & {$[-10.10]$} & 0 \\
\hline $\mathrm{f} 7=\sum_{i=1}^{D}\left|x_{i}\right|^{(i+1)}$ & {$[-1.28,1.28]$} & 0 \\
\hline $\mathrm{f} 8=\sum_{i=1}^{D} 10^{6 \frac{i-1}{D-1}}$ & {$[-1,1]$} & 0 \\
\hline $\mathrm{f} 9=\sum_{i=1}^{D}\left(\left|x_{i}+0.5\right|\right)$ & {$[-100,100]$} & 0 \\
\hline $\mathrm{f} 10=\sum_{i=1}^{D} i x_{i}^{4}+\operatorname{random}[0,1]$ & {$[-1.28,1.28]$} & 0 \\
\hline $\mathrm{f} 11=\sum_{i=1}^{D}\left(x_{i}^{2}-10 \cos \left(2 \pi x_{i}\right)+10\right)$ & {$[-5.12,5.12]$} & 0 \\
\hline $\begin{array}{l}\mathrm{f} 12=\mathrm{g} \\
-20 \exp \left(-0.2 * \sqrt{\sum_{i=1}^{D} x_{i}^{2}} / D\right)-\exp \left(\sum_{i=1}^{D} \cos (2 \pi x i / D)\right)+20+e\end{array}$ & {$[-32,32]$} & 0 \\
\hline
\end{tabular}


Table 1. Continued

\begin{tabular}{|c|c|c|}
\hline Functions & Range & $\begin{array}{c}\text { Optimal } \\
\text { Value }\end{array}$ \\
\hline $\mathrm{f} 13=\frac{1}{4000} \sum_{i=1}^{D} x_{i}^{2}-\prod_{i=1}^{n} \cos \left(\frac{z_{i}}{\sqrt{i}}\right)+1$ & {$[-600,600]$} & 0 \\
\hline $\mathrm{f} 14=0.5+\frac{\sin \left(\sqrt{\sum_{i=1}^{D} x_{i}^{2}}\right)^{2-0.5}}{\left(1+0.001 \sum_{i=1}^{D} x_{i}^{2}\right)^{2}}$ & {$[-100,100]$} & 0 \\
\hline $\mathrm{f} 15=\frac{\sum_{i=1}^{D}\left(x_{i}^{4}-16 x_{i}^{2}+5 x_{i}\right)}{D}$ & {$[-5,5]$} & 0 \\
\hline $\mathrm{f} 16=\sum_{i=1}^{D}\left|x_{i} \sin \left(x_{i}\right)+0.1 x_{i}\right|$ & {$[-10,10]$} & 0 \\
\hline $\begin{array}{l}\quad \sum_{i=1}^{D}\left(x_{i}^{2}-10 \cos \left(2 \pi x_{i}\right)+10\right) \quad\left|x_{i}<0\right| \\
\sum_{i=1}^{D}\left(\left(\frac{\operatorname{random}\left(2 x_{i}\right)}{2}\right)^{2}=10 \cos \left(\pi \operatorname{random} 2 x_{i}\right)\right)+10 \quad\left|x_{i} \geq 0.5\right|\end{array}$ & {$[-5.12,5.12]$} & 0 \\
\hline $\begin{array}{l}\mathrm{f} 18= \\
\frac{\pi}{D} 10 \sin ^{2}(\pi y 1)+\frac{\pi}{D} \sum_{i=1}^{D-1}(y i-1)^{2}\left[1+10 \sin ^{2}\left(\pi y_{i+1}\right)\right]+\frac{\pi}{D}\left(Y_{D}-1\right)^{2} \\
+\sum_{i=1}^{D} u\left(x_{i}, 10,100,4\right) y_{i}=1+\frac{x_{i+1}}{4} u\left(x_{i}, a, k, m\right) \\
=\left\{k\left(x_{i}-a\right)^{m}, x_{i}>a\right\} 0,-a \leq x_{i} \leq a k\left(-x_{i}-a\right)^{m}, x_{i}<-1\end{array}$ & {$[-50,50]$} & 0 \\
\hline $\mathrm{f} 19=\sum_{i=1}^{D}\left(20^{\frac{i-1}{D-1}} * z_{i}\right)^{2}, \mathrm{z}=\mathrm{x} * \mathrm{M}$ & {$[-100,100]$} & 0 \\
\hline 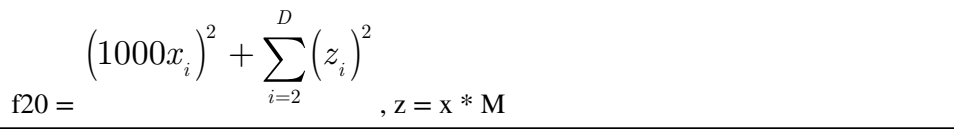 & {$[-100,100]$} & 0 \\
\hline $\mathrm{f} 21=\sum_{i=1}^{D} z_{i}^{2}, \mathrm{z}=\mathrm{x}-0$ & {$[-100,100]$} & 0 \\
\hline
\end{tabular}


Table 2. Results Obtained by pFA with different values of $\alpha 0$ and $\alpha 1$ on 30-dimensions

\begin{tabular}{|c|c|c|c|c|c|}
\hline Fun. & $\alpha 0=0.1, \alpha 1=0.25$ & $\alpha 0=0.3, \alpha 1=0.25$ & $\alpha 0=0.5, \alpha 1=0.25$ & $\alpha 0=0.7, \alpha 1=0.25$ & $\alpha 0=0.9, \alpha 1=0.25$ \\
\hline $\mathrm{F} 1$ & $0(0)$ & $0(0)$ & $0(0)$ & $0(0)$ & $1.03 e^{-224(0)}$ \\
\hline $\mathrm{F} 2$ & $1.59 e^{-280(0)}$ & $6.39 e^{-281(0)}$ & $1.80 e^{-281(0)}$ & $3.08 e^{-279(0)}$ & $\begin{array}{l}4.37 \\
e^{-113\left(4.58 e^{-113)}\right.}\end{array}$ \\
\hline F3 & $\begin{array}{l}1.78 \\
e^{-119\left(9.76 e^{-119)}\right.}\end{array}$ & $e^{-158\left(9.03 e^{-158}\right)}$ & $e^{6.35}$ & $e^{-133\left(9.06 e^{-133}\right)}$ & $\begin{array}{l}3.50 \\
e^{-113\left(4.58 e^{-113}\right)}\end{array}$ \\
\hline $\mathrm{F} 4$ & $2.66 \mathrm{e} e^{-242(0)}$ & $2.64 e^{-240(0)}$ & $9.00 e^{-243(0)}$ & $1.19 e^{-241(0)}$ & $\begin{array}{l}4.46 \\
e^{-113\left(5.38 e^{-114}\right)}\end{array}$ \\
\hline F5 & $0(0)$ & $0(0)$ & $0(0)$ & $0(0)$ & $1.55 e^{-225(0)}$ \\
\hline F6 & $0(0)$ & $0(0)$ & $0(0)$ & $0(0)$ & (0) \\
\hline F7 & $0(0)$ & $0(0)$ & $0(0)$ & $0(0)$ & $1.36 e^{-234(0)}$ \\
\hline F8 & $0(0)$ & $0(0)$ & $0(0)$ & $0(0)$ & $3.28 e^{-220(0)}$ \\
\hline F9 & $0(0)$ & $0(0)$ & $0(0)$ & $0(0)$ & $\begin{array}{l}2.2396 \\
e^{-63\left(1.1914 e^{-62)}\right.}\end{array}$ \\
\hline F10 & $1.06 e^{-05\left(1.02 e^{-05)}\right.}$ & $1.08 e^{-05\left(9.68 e^{-05)}\right.}$ & $1.39 e^{-05\left(1.88 e^{-05)}\right.}$ & $1.30 e^{-05\left(1.35 e^{-05)}\right.}$ & $8.94 e^{-06\left(9.51 e^{-06)}\right.}$ \\
\hline F11 & $0(0)$ & $0(0)$ & $0(0)$ & $0(0)$ & $0(0)$ \\
\hline F12 & $3.49 e^{-15\left(1.52 e^{-15}\right)}$ & $3.13 e^{-15\left(1.22 e^{-15)}\right.}$ & $3.37 e^{-15\left(1.44 e^{-15)}\right.}$ & $3.49 e^{-15\left(1.52 e^{-15)}\right.}$ & $8.94 e^{-15\left(3.45 e^{-15)}\right.}$ \\
\hline F13 & $0(0)$ & $0(0)$ & $0(0)$ & $0(0)$ & $0(0)$ \\
\hline F14 & $0.0097\left(6.23 e^{-11}\right)$ & $0.0097\left(3.41 e^{-07}\right)$ & $\begin{array}{l}0.0097(3.3167 \\
e^{-10)}\end{array}$ & $0.0097\left(2.16 e^{-07)}\right.$ & $0.0097\left(1.01 e^{-10)}\right.$ \\
\hline F15 & $-38.4619(2.0583)$ & $-39.0839(2.3885)$ & $-38.9468(2.2689)$ & $-39.52(2.4386)$ & $-46.8126(4.8447)$ \\
\hline F16 & $1.78 e^{-278(0)}$ & $1.97 e^{-280(0)}$ & $2.85 e^{-282(0)}$ & $1.21 e^{-282(0)}$ & $0.0034(0.0064)$ \\
\hline F17 & $0(0)$ & $0(0)$ & $0(0)$ & $0(0)$ & $0(0)$ \\
\hline F18 & $0.7996(0.2319)$ & $0.8171(0.2756)$ & $0.7627(2.2689)$ & $0.8048(0.3094)$ & $0.0468(0.0267)$ \\
\hline F19 & $1.86 e^{-12\left(1.02 e^{-11)}\right.}$ & $1.09 e^{-13\left(6.00 e^{-13)}\right.}$ & $5.46 e^{-12\left(2.52 e^{-11)}\right.}$ & $9.41 e^{-18\left(5.14 e^{-17)}\right.}$ & $4.06 e^{-15\left(2.18 e^{-14)}\right.}$ \\
\hline F20 & $0(0)$ & $0(0)$ & $0(0)$ & $0(0)$ & $2.28 e^{-221(0)}$ \\
\hline $\mathrm{F} 21$ & $0.0094\left(1.00 e^{-11)}\right.$ & $0.0110\left(1.21 e^{-10)}\right.$ & $0.0064\left(2.83 e^{-18)}\right.$ & $0.0108\left(9.11 e^{-19)}\right.$ & $0.0115\left(2.73 e^{-18)}\right.$ \\
\hline
\end{tabular}


Table 3. Results Obtained by pFA with different values of $\alpha 0$ and $\alpha 1$ on 30-dimensions

\begin{tabular}{|c|c|c|c|c|c|}
\hline Fun & $\alpha 0=0.1, \alpha 1=0.15$ & $\alpha 0=0.7, \alpha 1=0.35$ & $\alpha 0=0.7, \alpha 10.55$ & $\alpha 0=0.7, \alpha 1=0.75$ & $\alpha 0=0.7, \alpha 1=0.95$ \\
\hline $\mathrm{F} 1$ & $0(0)$ & $0(0)$ & $0(0)$ & $0(0)$ & $0(0)$ \\
\hline $\mathrm{F} 2$ & $7.80 e^{-280(0)}$ & $9.41 e^{-282(0)}$ & $8.86 e^{-279(0)}$ & $1.12 e^{-280(0)}$ & $4.37 e^{-280(0)}$ \\
\hline F3 & $e^{-127(5.08} e^{-126)}$ & $\begin{array}{l}8.944 \\
e^{-133\left(4.89 e^{-32)}\right.}\end{array}$ & $\begin{array}{l}1.16 \\
e^{-155\left(6.36 e^{-155)}\right.}\end{array}$ & $\begin{array}{l}9.98 \\
e^{-148\left(5.41 e^{-147)}\right.}\end{array}$ & $3.30 e^{-97\left(1.80 e^{-96)}\right.}$ \\
\hline F4 & $1.40 \mathrm{e} e^{-239(0)}$ & $7.76 e^{-242(0)}$ & $1.36 e^{-238(0)}$ & $1.41 e^{-241(0)}$ & $\begin{array}{l}4.87 \mathrm{a} \\
e^{-242\left(e^{-242(0)}\right.} .\end{array}$ \\
\hline F5 & $0(0)$ & $0(0)$ & $0(0)$ & $0(0)$ & $(0)$ \\
\hline F6 & $0(0)$ & $0(0)$ & $0(0)$ & 0) & (0) \\
\hline F7 & $0(0)$ & $0(0)$ & $0(0)$ & $0(0)$ & (0) \\
\hline F8 & $0(0)$ & $0(0)$ & $0(0)$ & $0(0)$ & (0) \\
\hline F9 & $0(0)$ & $0(0)$ & $0(0)$ & $0(0)$ & $0(0)$ \\
\hline F10 & $1.13 e^{-05\left(1.01 e^{-05)}\right.}$ & $9.88 e^{-06\left(1.06 e^{-05)}\right.}$ & $1.18 e^{-05\left(9.54 e^{-05)}\right.}$ & $1.04 e^{-05\left(9.87 e^{-05)}\right.}$ & $1.03 \mathrm{e}-05(1.27 \mathrm{e}-05)$ \\
\hline F11 & $0(0)$ & $0(0)$ & $0(0)$ & $0(0)$ & $0(0)$ \\
\hline F12 & $3.37 e^{-15\left(1.44 e^{-15)}\right.}$ & $3.84 e^{-15\left(1.70 e^{-15)}\right.}$ & $3.25 e^{-15\left(1.34 e^{-15)}\right.}$ & $3.49 e^{-15\left(1.52 e^{-15}\right)}$ & $3.49 \mathrm{e}-15(1.52 \mathrm{e}-15)$ \\
\hline F13 & $0(0)$ & $0(0)$ & $0(0)$ & $0(0)$ & $0(0)$ \\
\hline F14 & $0.0097\left(3.16 e^{-09}\right)$ & $0.0097\left(5.65 e^{-09)}\right.$ & $0.0097\left(1.65 e^{-08)}\right.$ & $0.0097\left(8.64 e^{-11)}\right.$ & $0.0097\left(1.01 e^{-11)}\right.$ \\
\hline $\mathrm{F} 15$ & $-39.0518(3.2473)$ & $-39.4378(2.0107)$ & $-38.7370(2.5830)$ & $-39.6825(2.8559)$ & $-39.1156(2.1769)$ \\
\hline F16 & $4.01 e^{-282(0)}$ & $1.27 e^{-280(0)}$ & $2.17 e^{-282(0)}$ & $1.38 e^{-282(0)}$ & $9.86(\mathrm{e}-282(0)$ \\
\hline F17 & $0(0)$ & $0(0)$ & $0(0)$ & $0(0)$ & $0(0)$ \\
\hline F18 & $0.7197(0.2319)$ & $0.7063(0.2046)$ & $0.7107(0.2567)$ & $0.7918(0.2931)$ & $0.7459(0.2802)$ \\
\hline F19 & $6.43 e^{-24\left(3.52 e^{-23)}\right.}$ & $1.42 e^{-22\left(7.82 e^{-22)}\right.}$ & $1.02 e^{-17\left(5.62 e^{-17)}\right.}$ & $9.87 e^{-12\left(5.39 e^{-11)}\right.}$ & $1.95 \mathrm{e}-20(1.06 \mathrm{e}-19)$ \\
\hline F20 & $0(0)$ & $0(0)$ & $1.13 e^{-17\left(5.62 e^{-17)}\right.}$ & $0(0)$ & $4.80 \mathrm{e}-09(9.40 \mathrm{e}-09)$ \\
\hline F21 & $0.0126\left(5.86 e^{-18)}\right.$ & $0.0122\left(4.89 e^{-18)}\right.$ & $0.0065\left(3.47 e^{-17)}\right.$ & $0.0146\left(5.06 e^{-18)}\right.$ & $0.0059\left(1.51 e^{-18)}\right.$ \\
\hline
\end{tabular}

$f_{19}$, pFA is able to converge to 0 within the iteration process, while others change little. For $f_{15}$, RaFA is better slightly than others, and for $f_{21}$, pFA is worse than others at the beginning, with the iteration goes, it quickly caught up with others. As shown in Figure 7 and Figure 8, as the dimension increase, 
International Journal of Cognitive Informatics and Natural Intelligence

Volume $14 \cdot$ Issue $3 \cdot$ July-September 2020

Table 4. Computational results of FA, RaFA, NaFA and pFA for each function on 30 dimensions

\begin{tabular}{|c|c|c|c|c|}
\hline Algorithm & Time & Min & Mean & Std \\
\hline $\begin{array}{l}\text { FA } \\
\text { RaFA } \\
\text { NaFA } \\
\text { pFA }\end{array}$ & $\begin{array}{l}249 \\
4 \\
6 \\
62\end{array}$ & $\begin{array}{l}0 \\
874.6161 \\
450.5938 \\
0\end{array}$ & $\begin{array}{l}0 \\
2.11 \mathrm{e}+03 \\
1.17 \mathrm{e}+03 \\
0\end{array}$ & $\begin{array}{l}0 \\
647.9445 \\
508.6607 \\
0\end{array}$ \\
\hline $\begin{array}{l}\text { FA } \\
\text { RaFA } \\
\text { NaFA } \\
\text { pFA }\end{array}$ & $\begin{array}{l}159 \\
4 \\
6 \\
69\end{array}$ & $\begin{array}{l}9.73 \mathrm{e}-322 \\
13.2200 \\
9.3405 \\
1.26 \mathrm{e}-292\end{array}$ & $\begin{array}{l}0.8723 \\
18.6540 \\
15.1840 \\
3.80 \mathrm{e}-279\end{array}$ & $\begin{array}{l}1.5287 \\
3.3840 \\
3.4537 \\
0\end{array}$ \\
\hline $\begin{array}{l}\text { FA } \\
\text { RaFA } \\
\text { NaFA } \\
\text { pFA }\end{array}$ & $\begin{array}{l}25 \\
4 \\
8 \\
93\end{array}$ & $\begin{array}{l}551.8415 \\
1.17 \mathrm{e}+03 \\
1.59 \mathrm{e}+03 \\
2.84 \mathrm{e}-273\end{array}$ & $\begin{array}{l}1.90 \mathrm{e}+03 \\
3.18 \mathrm{e}+03 \\
2.87 \mathrm{e}+03 \\
1.65 \mathrm{e}-133\end{array}$ & $\begin{array}{l}874.7558 \\
1.40 \mathrm{e}+03 \\
799.3186 \\
9.06 \mathrm{e}-133\end{array}$ \\
\hline $\begin{array}{l}\text { FA } \\
\text { RaFA } \\
\text { NaFA } \\
\text { pFA }\end{array}$ & $\begin{array}{l}25 \\
2 \\
5 \\
76\end{array}$ & $\begin{array}{l}0.0062 \\
15.4290 \\
10.3884 \\
1.57 \mathrm{e}-254\end{array}$ & $\begin{array}{l}2.9432 \\
20.0772 \\
16.2469 \\
1.19 \mathrm{e}-241\end{array}$ & $\begin{array}{l}2.5806 \\
3.4026 \\
2.7713 \\
0\end{array}$ \\
\hline $\begin{array}{l}\text { FA } \\
\text { RaFA } \\
\text { NaFA } \\
\text { pFA }\end{array}$ & $\begin{array}{l}22 \\
4 \\
6 \\
55\end{array}$ & $\begin{array}{l}1.76 \mathrm{e}-04 \\
174.1170 \\
62.8256 \\
0\end{array}$ & $\begin{array}{l}2.2007 \\
325.1527 \\
183.4959 \\
0\end{array}$ & $\begin{array}{l}4.2620 \\
77.6503 \\
69.9538 \\
0\end{array}$ \\
\hline $\begin{array}{l}\text { FA } \\
\text { RaFA } \\
\text { NaFA } \\
\text { pFA }\end{array}$ & $\begin{array}{l}164 \\
5 \\
8 \\
83\end{array}$ & $\begin{array}{l}0 \\
0.0233 \\
0.0025 \\
0\end{array}$ & $\begin{array}{l}0 \\
0.1905 \\
0.0998 \\
0\end{array}$ & $\begin{array}{l}0 \\
0.2088 \\
0.0747 \\
0\end{array}$ \\
\hline $\begin{array}{l}\text { FA } \\
\text { RaFA } \\
\text { NaFA } \\
\text { pFA }\end{array}$ & $\begin{array}{l}33 \\
5 \\
8 \\
83\end{array}$ & $\begin{array}{l}7.59 \mathrm{e}-08 \\
5.39 \mathrm{e}-08 \\
1.01 \mathrm{e}-08 \\
0\end{array}$ & $\begin{array}{l}1.13 \mathrm{e}-06 \\
5.39 \mathrm{e}-05 \\
2.19 \mathrm{e}-06 \\
0\end{array}$ & $\begin{array}{l}6.33 \mathrm{e}-07 \\
1.32 \mathrm{e}-04 \\
2.82 \mathrm{e}-06 \\
0\end{array}$ \\
\hline $\begin{array}{l}\text { FA } \\
\text { RaFA } \\
\text { NaFA } \\
\text { pFA }\end{array}$ & $\begin{array}{l}31 \\
5 \\
8 \\
107\end{array}$ & $\begin{array}{l}1.38 \mathrm{e}+06 \\
5.80 \mathrm{e}+06 \\
2.54 \mathrm{e}+06 \\
0\end{array}$ & $\begin{array}{l}4.91 \mathrm{e}+06 \\
3.26 \mathrm{e}+07 \\
9.51 \mathrm{e}+06 \\
0\end{array}$ & $\begin{array}{l}2.53 \mathrm{e}+06 \\
1.79 \mathrm{e}+07 \\
6.17 \mathrm{e}+06 \\
0\end{array}$ \\
\hline $\begin{array}{l}\text { FA } \\
\text { RaFA } \\
\text { NaFA } \\
\text { pFA }\end{array}$ & $\begin{array}{l}1 \\
0.5 \\
0.7 \\
44 \\
\end{array}$ & $\begin{array}{l}1 \\
1 \\
0 \\
0\end{array}$ & $\begin{array}{l}2.5330 \\
2.4333 \\
1.9000 \\
0\end{array}$ & $\begin{array}{l}1.3322 \\
1.1351 \\
1.1552 \\
0\end{array}$ \\
\hline $\begin{array}{l}\text { FA } \\
\text { RaFA } \\
\text { NaFA } \\
\text { pFA }\end{array}$ & $\begin{array}{l}1088 \\
31 \\
181 \\
125 \\
\end{array}$ & $\begin{array}{l}0.0193 \\
0.0742 \\
0.0705 \\
3.30 \mathrm{e}-08 \\
\end{array}$ & $\begin{array}{l}0.0552 \\
0.3144 \\
0.2063 \\
1.30 \mathrm{e}-05 \\
\end{array}$ & $\begin{array}{l}0.0250 \\
0.2032 \\
0.0900 \\
1.35 \mathrm{e}-05 \\
\end{array}$ \\
\hline $\begin{array}{l}\text { FA } \\
\text { RaFA } \\
\text { NaFA } \\
\text { pFA }\end{array}$ & $\begin{array}{l}63 \\
4 \\
10 \\
27 \\
\end{array}$ & $\begin{array}{l}31.8386 \\
101.9936 \\
65.0032 \\
0 \\
\end{array}$ & $\begin{array}{l}53.5950 \\
146.5316 \\
99.0897 \\
0 \\
\end{array}$ & $\begin{array}{l}12.5248 \\
21.3669 \\
16.2696 \\
0\end{array}$ \\
\hline $\begin{array}{l}\text { FA } \\
\text { RaFA } \\
\text { NaFA } \\
\text { pFA }\end{array}$ & $\begin{array}{l}22 \\
4 \\
6 \\
30\end{array}$ & $\begin{array}{l}6.21 \mathrm{e}-15 \\
7.3980 \\
5.5759 \\
2.66 \mathrm{e}-15\end{array}$ & $\begin{array}{l}1.67 \mathrm{e}-14 \\
9.5945 \\
8.2415 \\
3.49 \mathrm{e}-15\end{array}$ & $\begin{array}{l}5.31 \mathrm{e}-15 \\
0.9476 \\
1.1312 \\
1.52 \mathrm{e}-15\end{array}$ \\
\hline
\end{tabular}


Table 4. Continued

\begin{tabular}{|l|l|l|l|l|}
\hline \multicolumn{1}{|c|}{ Algorithm } & \multicolumn{1}{|c|}{ Time } & \multicolumn{1}{c|}{ Min } & \multicolumn{1}{c|}{ Mean } & \multicolumn{1}{c|}{ Std } \\
\hline FA & 27 & $5.02 \mathrm{e}-04$ & 0.8384 & 0.3028 \\
RaFA & 5 & 0.9594 & 0.9955 & 0.0076 \\
NaFA & 46 & 0.7904 & 0.9476 & 0.0485 \\
pFA & 35 & 0 & 0 & 0 \\
\hline FA & 21 & 0.1270 & 0.2915 & 0.0843 \\
RaFA & 3 & 0.4419 & 0.4735 & 0.0107 \\
NaFA & 59 & 0.4297 & 0.4706 & 0.0127 \\
pFA & 77 & 0.0097 & 0.0097 & $2.16 \mathrm{e}-07$ \\
\hline FA & 113 & -70.7927 & -67.3345 & 2.2962 \\
RaFA & 5 & -47.4986 & -41.3536 & 2.7055 \\
NaFA & 9 & -53.9663 & -48.5161 & 3.4484 \\
pFA & 123 & -45.8318 & -39.5200 & 2.4386 \\
\hline FA & 17 & 30 & 56.1333 & 17.7234 \\
RaFA & 5 & 66.8561 & 121.6540 & 23.2301 \\
NaFA & 17 & 40.4044 & 69.3322 & 13.0052 \\
pFA & 38 & 0 & 0 & 0 \\
\hline FA & 47 & $1.58 \mathrm{e}-27$ & 0.1424 & 0.3472 \\
RaFA & 7 & 5.3744 & 530.7299 & $2.30 \mathrm{e}+03$ \\
NaFA & 13 & 5.9968 & 15.1325 & 7.0716 \\
pFA & 182 & 0.3828 & 0.8048 & 0.3094 \\
\hline FA & 35 & $2.18 \mathrm{e}+03$ & $1.03 \mathrm{e}+04$ & $5.44 \mathrm{e}+03$ \\
RaFA & 6 & $3.07 \mathrm{e}+04$ & $5.82 \mathrm{e}+04$ & $2.04 \mathrm{e}+04$ \\
NaFA & 11 & $9.83 \mathrm{e}+03$ & $3.75 \mathrm{e}+04$ & $1.45 \mathrm{e}+04$ \\
pFA & 135 & 0 & $9.41 \mathrm{e}-18$ & $5.14 \mathrm{e}-17$ \\
\hline FA & 27 & $1.58 \mathrm{e}+04$ & $2.63 \mathrm{e}+04$ & $7.21 \mathrm{e}+03$ \\
RaFA & 4 & $2.27 \mathrm{e}+03$ & $6.05 \mathrm{e}+03$ & $2.27 \mathrm{e}+03$ \\
NaFA & 8 & $3.44 \mathrm{e}+03$ & $6.76 \mathrm{e}+03$ & $2.40 \mathrm{e}+03$ \\
pFA & 78 & 0 & 0 & 0 \\
\hline FA & 30 & 0.0173 & 0.0173 & $3.34 \mathrm{e}-18$ \\
RaFA & 36 & 0.0131 & 0.0131 & $8.38 \mathrm{e}-18$ \\
NaFA & 78 & 0.0137 & 0.0137 & $6.54 \mathrm{e}-18$ \\
pFA & & 0.0108 & $9.11 \mathrm{e}-19$ \\
\hline
\end{tabular}

the convergence of pFA becomes slower in $f_{3}$. For $f_{7}, f_{11}$ and $f_{21}$ all of them remain unchanged, and for $f 15$ and $f 19$, pFA becomes better, while others change little.

In summary, the performance of pFA is better than FA, RaFA and NaFA on most functions, and for majority functions, pFA can find the minimum value of 0 and it also effectively for solving higher dimension problem.

\section{Experiment 3: Comparison With Other FA's Basedon a Probabilistic Metric}

In (Yu et al., 2015), Gomes et al. proposed a new way of comparison among metaheuristic optimization algorithms, in this way, it can be clearly seen which algorithm is better. In this paper, as shown in (Yu et al., 2015), we regard $P_{\text {better }}$ as the probability that represents pFA is better than FA, RaFA and NaFA. If pFA is worse than FA, RaFA and NaFA, we regard $P_{\text {better }}$ as the probability, and if pFA is equal to FA, RaFA and NaFA, we regard $P_{\text {equal }}$ as the probability. Here, the population size, dimension and the maximum iterations are set to 40,50 and 2500 , respectively. Moreover, we select $f_{3}, f_{7}, f_{11}, f_{15}$, $f_{19}$ and $f_{21}$ as benchmark functions, and for each algorithm, it was run 30 times. The results are shown in Table 7. As shown in Table 7, for $f_{3}, f_{7}, f_{11}$, and $f_{19}$, pFA is the best, and it is $100 \%$ likely to find 
International Journal of Cognitive Informatics and Natural Intelligence Volume $14 \cdot$ Issue $3 \cdot$ July-September 2020

Table 5. Computational results of FA, RaFA, NaFA, and pFA for each function on 30 dimensions

\begin{tabular}{|c|c|c|c|c|c|}
\hline Function & Algorithm & Time & Min & Mean & Std. \\
\hline $\mathrm{F} 1$ & $\begin{array}{l}\text { FA } \\
\text { RaFA } \\
\text { NaFA } \\
\text { pFA }\end{array}$ & $\begin{array}{l}49 \\
5 \\
7 \\
68\end{array}$ & $\begin{array}{l}3.03 e-104 \\
3.26 e+03 \\
2.67 e+03 \\
0\end{array}$ & $\begin{array}{l}1.60 \mathrm{e}-04 \\
5.15 \mathrm{e}+03 \\
4.49 \mathrm{e}+03 \\
0\end{array}$ & $\begin{array}{l}6.17 e-14 \\
1.83 e+03 \\
1.08 e+03 \\
0\end{array}$ \\
\hline $\mathrm{F} 2$ & $\begin{array}{l}\text { FA } \\
\text { RaFA } \\
\text { NaFA } \\
\text { pFA }\end{array}$ & $\begin{array}{l}23 \\
4 \\
6 \\
74\end{array}$ & $\begin{array}{l}1.0787 \\
29.6556 \\
27.7283 \\
3.31 \mathrm{e}-277\end{array}$ & $\begin{array}{l}13.9119 \\
39.2844 \\
38.6810 \\
1.47 \mathrm{e}-266\end{array}$ & $\begin{array}{l}8.7174 \\
4.1969 \\
6.0088 \\
0\end{array}$ \\
\hline $\mathrm{F} 3$ & $\begin{array}{l}\text { FA } \\
\text { RaFA } \\
\text { NaFA } \\
\text { pFA }\end{array}$ & $\begin{array}{l}34 \\
6 \\
12 \\
129\end{array}$ & $\begin{array}{l}5.41 \mathrm{e}+03 \\
4.09 \mathrm{e}+03 \\
5.60 \mathrm{e}+03 \\
5.39 \mathrm{e}-179\end{array}$ & $\begin{array}{l}9.71 \mathrm{e}+03 \\
1.06 \mathrm{e}+04 \\
9.23 \mathrm{e}+03 \\
5.12 \mathrm{e}-62\end{array}$ & $\begin{array}{l}3.07 \mathrm{e}+03 \\
4.58 \mathrm{e}+03 \\
2.80 \mathrm{e}+03 \\
2.80 \mathrm{e}-61\end{array}$ \\
\hline $\mathrm{F} 4$ & $\begin{array}{l}\text { FA } \\
\text { RaFA } \\
\text { NaFA } \\
\text { pFA }\end{array}$ & $\begin{array}{l}26 \\
3 \\
6 \\
83\end{array}$ & $\begin{array}{l}7.2956 \\
18.3396 \\
18.2441 \\
4.44 \mathrm{e}-242\end{array}$ & $\begin{array}{l}14.0661 \\
23.7321 \\
22.5221 \\
2.41 \mathrm{e}-234\end{array}$ & $\begin{array}{l}2.8475 \\
2.9446 \\
2.6046 \\
0\end{array}$ \\
\hline F5 & $\begin{array}{l}\text { FA } \\
\text { RaFA } \\
\text { NaFA } \\
\text { pFA }\end{array}$ & $\begin{array}{l}22 \\
4 \\
6 \\
65\end{array}$ & $\begin{array}{l}3.8608 \\
726.9976 \\
585.9786 \\
0\end{array}$ & $\begin{array}{l}42.1408 \\
1.47 \mathrm{e}+03 \\
1.06 \mathrm{e}+03 \\
0\end{array}$ & $\begin{array}{l}29.4447 \\
260.9366 \\
316.2513 \\
0\end{array}$ \\
\hline F6 & $\begin{array}{l}\text { FA } \\
\text { RaFA } \\
\text { NaFA } \\
\text { pFA }\end{array}$ & $\begin{array}{l}41 \\
6 \\
10 \\
118\end{array}$ & $\begin{array}{l}2.51 \mathrm{e}-09 \\
0.2298 \\
0.2569 \\
0\end{array}$ & $\begin{array}{l}1.27 \mathrm{e}-04 \\
0.9258 \\
0.7123 \\
0\end{array}$ & $\begin{array}{l}1.63 \mathrm{e}-04 \\
0.4317 \\
0.3796 \\
0\end{array}$ \\
\hline F7 & $\begin{array}{l}\text { FA } \\
\text { RaFA } \\
\text { NaFA } \\
\text { pFA }\end{array}$ & $\begin{array}{l}41 \\
6 \\
9 \\
109\end{array}$ & $\begin{array}{l}2.17 \mathrm{e}-07 \\
6.67 \mathrm{e}-08 \\
1.70 \mathrm{e}-09 \\
0\end{array}$ & $\begin{array}{l}1.34 \mathrm{e}-06 \\
8.38 \mathrm{e}-05 \\
3.98 \mathrm{e}-06 \\
0\end{array}$ & $\begin{array}{l}8.20 \mathrm{e}-07 \\
2.55 \mathrm{e}-04 \\
4.54 \mathrm{e}-06 \\
0\end{array}$ \\
\hline F8 & $\begin{array}{l}\mathrm{Fa} \\
\mathrm{RaFA} \\
\mathrm{NaFA} \\
\text { pFA }\end{array}$ & $\begin{array}{l}38 \\
6 \\
10 \\
150\end{array}$ & $\begin{array}{l}6.40 \mathrm{e}+06 \\
3.92 \mathrm{e}+07 \\
1.12 \mathrm{e}+07 \\
0\end{array}$ & $\begin{array}{l}1.81 \mathrm{e}+07 \\
1.06 \mathrm{e}+08 \\
4.31 \mathrm{e}+07 \\
0\end{array}$ & $\begin{array}{l}8.36 \mathrm{e}+06 \\
5.49 \mathrm{e}+07 \\
1.94 \mathrm{e}+07 \\
0\end{array}$ \\
\hline F10 & $\begin{array}{l}\text { FA } \\
\text { RaFA } \\
\text { NaFA } \\
\text { pFA }\end{array}$ & $\begin{array}{l}1422 \\
38 \\
230 \\
168\end{array}$ & $\begin{array}{l}0.0918 \\
0.3719 \\
0.4187 \\
3.44 \mathrm{e}-07\end{array}$ & $\begin{array}{l}0.8179 \\
1.0542 \\
1.0951 \\
8.09 \mathrm{e}-06\end{array}$ & $\begin{array}{l}0.0638 \\
0.5028 \\
0.5019 \\
7.92 \mathrm{e}-06\end{array}$ \\
\hline F11 & $\begin{array}{l}\text { FA } \\
\text { RaFA } \\
\text { NaFA } \\
\text { pFA }\end{array}$ & $\begin{array}{l}44 \\
4 \\
8 \\
31\end{array}$ & $\begin{array}{l}60.6924 \\
238.4711 \\
180.3972 \\
0\end{array}$ & $\begin{array}{l}100.9517 \\
300.2502 \\
246.0055 \\
0\end{array}$ & $\begin{array}{l}21.1218 \\
31.4020 \\
24.5482 \\
0\end{array}$ \\
\hline F13 & $\begin{array}{l}\text { FA } \\
\text { RaFA } \\
\text { NaFA } \\
\text { pFA }\end{array}$ & $\begin{array}{l}20 \\
4 \\
39 \\
44\end{array}$ & $\begin{array}{l}1.0000 \\
1.0000 \\
0.9937 \\
0\end{array}$ & $\begin{array}{l}1.0000 \\
1.0000 \\
0.9994 \\
0\end{array}$ & $\begin{array}{l}7.26 \mathrm{e}-10 \\
1.48 \mathrm{e}-06 \\
0.0014 \\
0\end{array}$ \\
\hline $\mathrm{F} 14$ & $\begin{array}{l}\text { FA } \\
\text { RaFA } \\
\text { NaFA } \\
\text { pFA }\end{array}$ & $\begin{array}{l}73 \\
3 \\
64 \\
82\end{array}$ & $\begin{array}{l}0.4147 \\
0.4850 \\
0.4888 \\
0.0097\end{array}$ & $\begin{array}{l}0.4798 \\
0.4930 \\
0.4932 \\
0.0097\end{array}$ & $\begin{array}{l}0.0177 \\
0.0031 \\
0.0023 \\
6.87 \mathrm{e}-08\end{array}$ \\
\hline
\end{tabular}




\begin{tabular}{|l|l|l|l|l|l|}
\hline Function & \multicolumn{1}{|c|}{ Algorithm } & \multicolumn{1}{c|}{ Time } & \multicolumn{1}{c|}{ Min } & \multicolumn{1}{c|}{ Mean } & \multicolumn{1}{c|}{ Std. } \\
\hline \multirow{5}{*}{ F15 } & FA & 38 & -70.1781 & -66.0138 & 1.9884 \\
& RaFA & 7 & -40.9722 & -37.7798 & 1.8800 \\
& NaFA & 13 & -48.9140 & -43.6976 & 2.6474 \\
& pFA & 167 & -41.4030 & -35.9725 & 2.5076 \\
\hline \multirow{5}{*}{ F16 } & FA & 28 & 0.7043 & 3.0358 & 1.6669 \\
& RaFA & 4 & 18.9912 & 25.1443 & 2.7943 \\
& NaFA & 8 & 16.6327 & 20.4971 & 2.5943 \\
& pFA & 76 & $2.20 \mathrm{e}-277$ & $1.43 \mathrm{e}-266$ & 0 \\
\hline \multirow{5}{*}{ F17 } & FA & 18 & 68 & 111.9750 & 28.0144 \\
& RaFA & 5 & 193.7580 & 258.1913 & 29.0084 \\
& NaFA & 23 & 150.2852 & 189.0966 & 23.2016 \\
& pFA & 43 & 0 & 0 & 0 \\
\hline \multirow{5}{*}{ F19 } & FA & 54 & 1.0212 & 4.6927 & 2.1361 \\
& RaFA & 9 & 9.7041 & $6.15 \mathrm{e}+03$ & $2.20 \mathrm{e}+04$ \\
& NaFA & 15 & 15.5344 & $1.14 \mathrm{e}+03$ & $4.19 \mathrm{e}+03$ \\
& pFA & 241 & 0.51454 & 0.8954 & 0.2381 \\
\hline \multirow{5}{*}{ F20 } & FA & 45 & $1.26 \mathrm{e}+0.4$ & $4.59 \mathrm{e}+04$ & $2.28 \mathrm{e}+04$ \\
& RaFA & 8 & $8.21 \mathrm{e}+04$ & $1.73 \mathrm{e}+05$ & $6.05 \mathrm{e}+04$ \\
& NaFA & 16 & $6.17 \mathrm{e}+04$ & $1.23 \mathrm{e}+05$ & $3.69 \mathrm{e}+04$ \\
& $\mathrm{pFA}$ & 195 & 0 & $1.95 \mathrm{e}-66$ & $1.07 \mathrm{e}-65$ \\
\hline \multirow{5}{*}{} & FA & 30 & $2.98 \mathrm{e}+04$ & $4.84 \mathrm{e}+04$ & $1.29 \mathrm{e}+04$ \\
& RaFA & 5 & $6.51 \mathrm{e}+03$ & $1.05 \mathrm{e}+04$ & $2.43 \mathrm{e}+03$ \\
& NaFA & 9 & $7.90 \mathrm{e}+03$ & $1.36 \mathrm{e}+04$ & $3.53 \mathrm{e}+03$ \\
& pFA & 92 & 0 & 0 & 0 \\
\hline & FA & 74 & 0.0091 & 0.0091 & $3.42 \mathrm{e}-18$ \\
& RaFA & 19 & 0.0073 & 0.0073 & $4.39 \mathrm{e}-18$ \\
& NaFA & 93 & 0.0062 & 0.0062 & $2.24 \mathrm{e}-18$ \\
& Pfa & 113 & 0.0059 & 0.0059 & $2.95 \mathrm{e}-18$ \\
\hline
\end{tabular}

the optimal value in a single run. For $f_{15}$, it can be seen that pFA is worse than FA, RaFA and NaFA with the probability of $100 \%, 72 \%$ and $99 \%$, respectively. And for $f_{21}$, pFA is better than FA, RaFA and NaFA with the probability of $100 \%$.

\section{Experiment 4: Comparison With De and DeSH}

In this part, the validity of the improved algorithm is furtherproved by comparing with DE, chDE (Zhenyu et al., 2006), jDE (Brest et al., 2006), aDE (Noman et al., 2011) and IMMSADE (Wang et al., 2017) on $f_{1}-f_{9}, f_{11}, f_{13}, f_{15}, f_{17}, f_{19}$, and $f_{21}$. These benchmark functions are consistent with literature. For the sake of fairness, all parameter settings are derived from literature. For each algorithm, program runs 30 times independently. The dimension $D$ and the population size $N P$ are set to 30, 100, respectively, and the maximum iterations (ItMax) is set to 3000. We utilize the statistics of average value (mean) and standard deviation (std) to evaluate the performance of algorithms after 30 times running. The statistical results are displayed in Table 7. And except for the statistics of pFA, statistics of others are derived from their original papers. For example, the parameter settings of IMMSADE algorithm are the same as literature (Wang et al., 2017). Besides, the parameter settings of DE originate from literature, in Which $C R$ is set to 0.9 and $F$ is set to $0.9,0.5$. For pFA, $\beta_{0}$, and are set to 1 .

As shown in Table 8, for $f 1, f 3-f 7$ and $f 9$, both mean and std of pFA are superior to others'. In particular, both of them are 0 on $f 1, f 3, f 6$ and $f 7$. For $f 8$ and $f 15$, the values of mean and std of all algorithms are 0 . For $f 13$, pFA is as good as aDE and IMMSADE, of which mean and std are0. 
International Journal of Cognitive Informatics and Natural Intelligence Volume $14 \cdot$ Issue $3 \cdot$ July-September 2020

Table 6. Computational results of FA, RaFA, NaFA, and pFA for each function on 100 dimensions

\begin{tabular}{|c|c|c|c|c|c|}
\hline Function & Algorithm & Time & Min & Mean & Std. \\
\hline $\mathrm{F} 1$ & $\begin{array}{l}\text { FA } \\
\text { RaFA } \\
\text { NaFA } \\
\text { Pfa }\end{array}$ & $\begin{array}{l}28 \\
5 \\
8 \\
79\end{array}$ & $\begin{array}{l}1.25 \mathrm{e}+03 \\
1.05 \mathrm{e}+04 \\
1.24 \mathrm{e}+04 \\
0\end{array}$ & $\begin{array}{l}3.28 \mathrm{e}+03 \\
1.39 \mathrm{e}+04 \\
1.73 \mathrm{e}+04 \\
0\end{array}$ & $\begin{array}{l}1.61 e+03 \\
2.04 e+03 \\
2.75 e+03 \\
0\end{array}$ \\
\hline $\mathrm{F} 2$ & $\begin{array}{l}\text { FA } \\
\text { RaFA } \\
\text { NaFA } \\
\text { Pfa }\end{array}$ & $\begin{array}{l}25 \\
4 \\
7 \\
83\end{array}$ & $\begin{array}{l}45.7986 \\
75.6588 \\
86.5001 \\
1.51 \mathrm{e}-265\end{array}$ & $\begin{array}{l}93.6254 \\
91.2161 \\
105.3091 \\
7.46 \mathrm{e}-265\end{array}$ & $\begin{array}{l}19.3097 \\
8.1710 \\
9.7488 \\
0\end{array}$ \\
\hline $\mathrm{F} 3$ & $\begin{array}{l}\text { FA } \\
\text { RaFA } \\
\text { NaFA } \\
\text { Pfa }\end{array}$ & $\begin{array}{l}33 \\
6 \\
13 \\
129\end{array}$ & $\begin{array}{l}6.76 \mathrm{e}+03 \\
5.97 \mathrm{e}- \\
3.91 \mathrm{e}- \\
8.96 \mathrm{e}-\end{array}$ & $\begin{array}{l}9.85 \mathrm{e}+03 \\
9.63 \mathrm{e}+03 \\
9.63 \mathrm{e}+03 \\
1.32 \mathrm{e}-56\end{array}$ & $\begin{array}{l}2.40 \mathrm{e}+03 \\
3.22 \mathrm{e}+03 \\
3.22 \mathrm{e}+03 \\
6.58 \mathrm{e}-59\end{array}$ \\
\hline $\mathrm{F} 4$ & $\begin{array}{l}\text { FA } \\
\text { RaFA } \\
\text { NaFA } \\
\text { Pfa }\end{array}$ & $\begin{array}{l}29 \\
3 \\
6 \\
91\end{array}$ & $\begin{array}{l}19.70 \\
22.96 \\
25.58 \\
6.78 \mathrm{e}-\end{array}$ & $\begin{array}{l}29.5103 \\
29.3519 \\
30.6770 \\
2.67 \mathrm{e}-227\end{array}$ & $\begin{array}{l}3.3598 \\
2.7539 \\
2.2555 \\
0\end{array}$ \\
\hline F5 & $\begin{array}{l}\text { FA } \\
\text { RaFA } \\
\text { NaFA } \\
\text { Pfa }\end{array}$ & $\begin{array}{l}25 \\
4 \\
7 \\
74\end{array}$ & $\begin{array}{l}724.73 \\
4.13 \mathrm{e}- \\
5.13 \mathrm{e}- \\
0\end{array}$ & $\begin{array}{l}2.04 \mathrm{e}+03 \\
6.54 \mathrm{e}+03 \\
7.82 \mathrm{e}+03 \\
0\end{array}$ & $\begin{array}{l}716.0177 \\
1.19 \mathrm{e}+03 \\
1.22 \mathrm{e}+03 \\
0\end{array}$ \\
\hline F6 & $\begin{array}{l}\text { FA } \\
\text { RaFA } \\
\text { NaFA } \\
\text { Pfa }\end{array}$ & $\begin{array}{l}56 \\
9 \\
16 \\
197\end{array}$ & $\begin{array}{l}0.254 \\
3.013 \\
4.019 \\
0\end{array}$ & $\begin{array}{l}1.1921 \\
6.3736 \\
9.5038 \\
0\end{array}$ & $\begin{array}{l}0.6860 \\
2.0038 \\
2.7436 \\
0\end{array}$ \\
\hline F7 & $\begin{array}{l}\text { FA } \\
\text { RaFA } \\
\text { NaFA } \\
\text { Pfa }\end{array}$ & $\begin{array}{l}60 \\
8 \\
14 \\
177\end{array}$ & $\begin{array}{l}4.56 \mathrm{e}- \\
1.53 \mathrm{e}- \\
2.94 \mathrm{e}- \\
0\end{array}$ & $\begin{array}{l}1.41 \mathrm{e}-06 \\
2.14 \mathrm{e}-04 \\
3.41 \mathrm{e}-06 \\
0\end{array}$ & $\begin{array}{l}7.20 \mathrm{e}-07 \\
8.78 \mathrm{e}-04 \\
5.64 \mathrm{e}-06 \\
0\end{array}$ \\
\hline F8 & $\begin{array}{l}\text { FA } \\
\text { RaFA } \\
\text { NaFA } \\
\text { Pfa }\end{array}$ & $\begin{array}{l}55 \\
9 \\
17 \\
260\end{array}$ & $\begin{array}{l}3.44 \mathrm{e}- \\
1.70 \mathrm{e}- \\
9.24 \mathrm{e}- \\
0\end{array}$ & $\begin{array}{l}8.77 \mathrm{e}+07 \\
3.71 \mathrm{e}+08 \\
2.51 \mathrm{e}+08 \\
0\end{array}$ & $\begin{array}{l}2.27 e+07 \\
1.13 e+07 \\
9.16 e+07 \\
0\end{array}$ \\
\hline F9 & $\begin{array}{l}\text { FA } \\
\text { RaFA } \\
\text { NaFA } \\
\text { Pfa }\end{array}$ & $\begin{array}{l}2 \\
0.8 \\
1.3 \\
63\end{array}$ & $\begin{array}{l}9 \\
19 \\
15 \\
4\end{array}$ & $\begin{array}{l}17.3333 \\
24.5667 \\
21.2000 \\
8.2000\end{array}$ & $\begin{array}{l}4.1133 \\
3.5006 \\
2.4691 \\
2.3839\end{array}$ \\
\hline F10 & $\begin{array}{l}\text { FA } \\
\text { RaFA } \\
\text { NaFA } \\
\text { Pfa }\end{array}$ & $\begin{array}{l}2086 \\
56 \\
349 \\
274\end{array}$ & $\begin{array}{l}1.1660 \\
3.9583 \\
4.7223 \\
1.044 \mathrm{e}-07\end{array}$ & $\begin{array}{l}3.0710 \\
7.2508 \\
11.4290 \\
1.40 \mathrm{e}-05\end{array}$ & $\begin{array}{l}1.2766 \\
3.0172 \\
4.7380 \\
1.43 \mathrm{e}-05\end{array}$ \\
\hline F11 & $\begin{array}{l}\text { FA } \\
\text { RaFA } \\
\text { NaFA } \\
\text { Pfa }\end{array}$ & $\begin{array}{l}29 \\
5 \\
10 \\
39\end{array}$ & $\begin{array}{l}182.9112 \\
666.9288 \\
573.5190 \\
0\end{array}$ & $\begin{array}{l}283.1775 \\
743.7117 \\
643.1476 \\
0\end{array}$ & $\begin{array}{l}32.5341 \\
43.0929 \\
30.7975 \\
0\end{array}$ \\
\hline $\mathrm{F} 12$ & $\begin{array}{l}\text { FA } \\
\text { RaFA } \\
\text { NaFA } \\
\text { Pfa }\end{array}$ & $\begin{array}{l}28 \\
5 \\
11 \\
38\end{array}$ & $\begin{array}{l}6.2005 \\
11.2087 \\
11.0521 \\
2.66 \mathrm{e}-15\end{array}$ & $\begin{array}{l}8.4004 \\
12.1025 \\
12.9293 \\
4.08 \mathrm{e}-15\end{array}$ & $\begin{array}{l}1.1137 \\
0.4671 \\
0.6050 \\
1.77 \mathrm{e}-15\end{array}$ \\
\hline
\end{tabular}


Table 6. Continued

\begin{tabular}{|c|c|c|c|c|c|}
\hline Function & Algorithm & Time & Min & Mean & Std. \\
\hline F13 & $\begin{array}{l}\text { FA } \\
\text { RaFA } \\
\text { NaFA } \\
\text { Pfa }\end{array}$ & $\begin{array}{l}27 \\
1 \\
5 \\
61\end{array}$ & $\begin{array}{l}1.0000 \\
1.0000 \\
1.0000 \\
0\end{array}$ & $\begin{array}{l}1.0000 \\
1.0000 \\
1.0000 \\
0\end{array}$ & $\begin{array}{l}0 \\
0 \\
0 \\
0\end{array}$ \\
\hline F14 & $\begin{array}{l}\text { FA } \\
\text { RaFA } \\
\text { NaFA } \\
\text { Pfa }\end{array}$ & $\begin{array}{l}115 \\
4 \\
71 \\
93\end{array}$ & $\begin{array}{l}0.4979 \\
0.4980 \\
0.4976 \\
0.0097\end{array}$ & $\begin{array}{l}0.4991 \\
0.4988 \\
0.4987 \\
0.0097\end{array}$ & $\begin{array}{l}4.12 \mathrm{e}-04 \\
3.49 \mathrm{e}-04 \\
3.83 \mathrm{e}-04 \\
1.78 \mathrm{e}-08\end{array}$ \\
\hline F15 & $\begin{array}{l}\text { FA } \\
\text { RaFA } \\
\text { NaFA } \\
\text { Pfa }\end{array}$ & $\begin{array}{l}58 \\
12 \\
23 \\
270\end{array}$ & $\begin{array}{l}-62.4677 \\
-38.9661 \\
-43.6207 \\
-35.8802\end{array}$ & $\begin{array}{l}-59.1327 \\
-34.5243 \\
-37.4587 \\
-33.1229\end{array}$ & $\begin{array}{l}1.7 .38 \\
1.7015 \\
2.0631 \\
1.4444\end{array}$ \\
\hline F16 & $\begin{array}{l}\text { FA } \\
\text { RaFA } \\
\text { NaFA } \\
\text { Pfa }\end{array}$ & $\begin{array}{l}28 \\
5 \\
9 \\
90\end{array}$ & $\begin{array}{l}12.8347 \\
51.0554 \\
52.8338 \\
1.79 \mathrm{e}-265\end{array}$ & $\begin{array}{l}22.8740 \\
61.2341 \\
60.5799 \\
2.50 \mathrm{e}-257\end{array}$ & $\begin{array}{l}4.6885 \\
4.7078 \\
4.9688 \\
0\end{array}$ \\
\hline F17 & $\begin{array}{l}\text { FA } \\
\text { RaFA } \\
\text { NaFA } \\
\text { Pfa }\end{array}$ & $\begin{array}{l}27 \\
5 \\
20 \\
55\end{array}$ & $\begin{array}{l}234.5053 \\
575.2118 \\
477.7268 \\
0\end{array}$ & $\begin{array}{l}322.2796 \\
672.3144 \\
546.7777 \\
0\end{array}$ & $\begin{array}{l}46.2017 \\
46.8110 \\
36.9873 \\
0\end{array}$ \\
\hline F19 & $\begin{array}{l}\text { FA } \\
\text { RaFA } \\
\text { NaFA } \\
\text { Pfa } \\
\end{array}$ & $\begin{array}{l}78 \\
14 \\
26 \\
362 \\
\end{array}$ & $\begin{array}{l}1.29 \mathrm{e}+05 \\
3.45 \mathrm{e}+05 \\
3.82 \mathrm{e}+05 \\
0\end{array}$ & $\begin{array}{l}2.36 \mathrm{e}+05 \\
5.29 \mathrm{e}+05 \\
5.66 \mathrm{e}+05 \\
1.95 \mathrm{e}-66\end{array}$ & $\begin{array}{l}5.19 \mathrm{e}+04 \\
1.11 \mathrm{e}+05 \\
1.22 \mathrm{e}+05 \\
1.07 \mathrm{e}-65 \\
\end{array}$ \\
\hline F20 & $\begin{array}{l}\text { FA } \\
\text { RaFA } \\
\text { NaFA } \\
\text { Pfa } \\
\end{array}$ & $\begin{array}{l}45 \\
7 \\
15 \\
152 \\
\end{array}$ & $\begin{array}{l}6.43 e+04 \\
1.75 e+04 \\
2.16 e+04 \\
0\end{array}$ & $\begin{array}{l}9.95 \mathrm{e}+04 \\
2.45 \mathrm{e}+04 \\
2.91 \mathrm{e}+04 \\
0\end{array}$ & $\begin{array}{l}2.72 \mathrm{e}+04 \\
5.03 \mathrm{e}+03 \\
4.63 \mathrm{e}+03 \\
0\end{array}$ \\
\hline $\mathrm{F} 21$ & $\begin{array}{l}\text { FA } \\
\text { RaFA } \\
\text { NaFA } \\
\text { Pfa }\end{array}$ & $\begin{array}{l}165 \\
29 \\
217 \\
210\end{array}$ & $\begin{array}{l}0.0038 \\
0.0037 \\
0.0033 \\
0.0031\end{array}$ & $\begin{array}{l}0.0038 \\
0.0037 \\
0.0033 \\
0.0031\end{array}$ & $\begin{array}{l}1.62 \mathrm{e}-18 \\
1.93 \mathrm{e}-18 \\
1.15 \mathrm{e}-18 \\
2.02 \mathrm{e}-18\end{array}$ \\
\hline
\end{tabular}

For $f 2$ and $f 17$, the performance of pFA is worse than others'. Furthermore, mean and std of pFA is slightly lower than others' in $f 21$. In conclusion, the results show that pFA is reliable and effective.

\section{SCALE-INVARIANT FEATURE TRANSFORM}

Harris feature detectors are rotation invariant i.e. same corners in images are detected even after image rotation and before rotation, but it fails to detect corners when image is scaled. After scaling the image, corners in an image may or may not be corners. So, this feature detector is scale variant Liang \& Mendel, 2000). To overcome this drawback, scale-invariant feature transform (SIFT) is introduced in the year 2004. The algorithm follows 4 steps:

\section{Scale Space Peak Selection: Potential Locations for Finding Features}

Different sizes of windows are used for different scaled images for detection of key points and this process is known as scale space filtering, difference of Laplacian of Gaussian (DoG) produce windows of different sizes. Difference of Gaussian value is calculated by difference of two Gaussian images. 
Figure 6. Convergence curve of $f_{3}, f_{7}, f_{11}, f_{15}, f_{19}$ and $f_{21}$ on 30 dimension
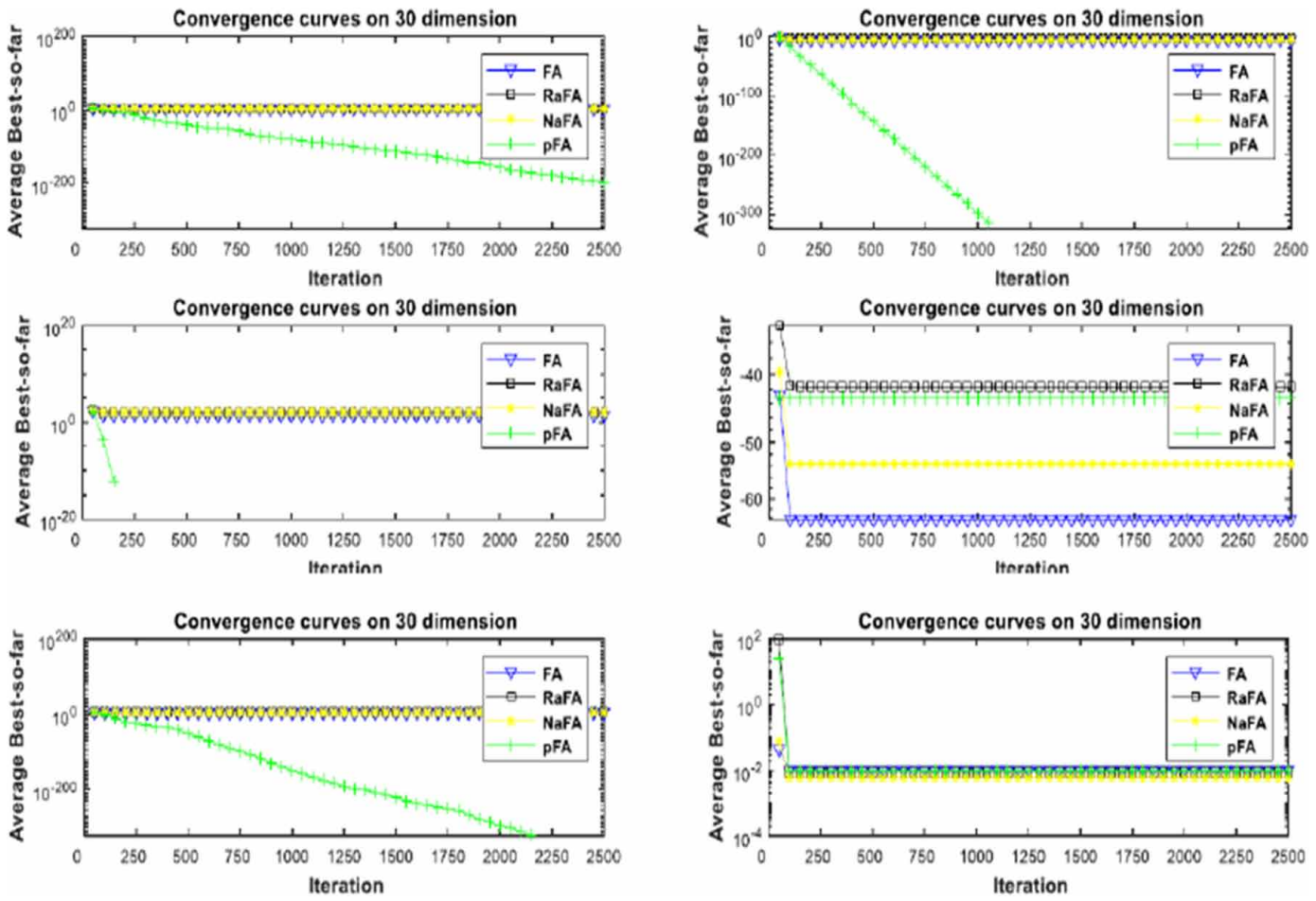

Figure 7. Convergence curve of $f_{3}, f_{7}, f_{11}, f_{15}, f_{19}$, and $f_{21}$ on 50 dimensions
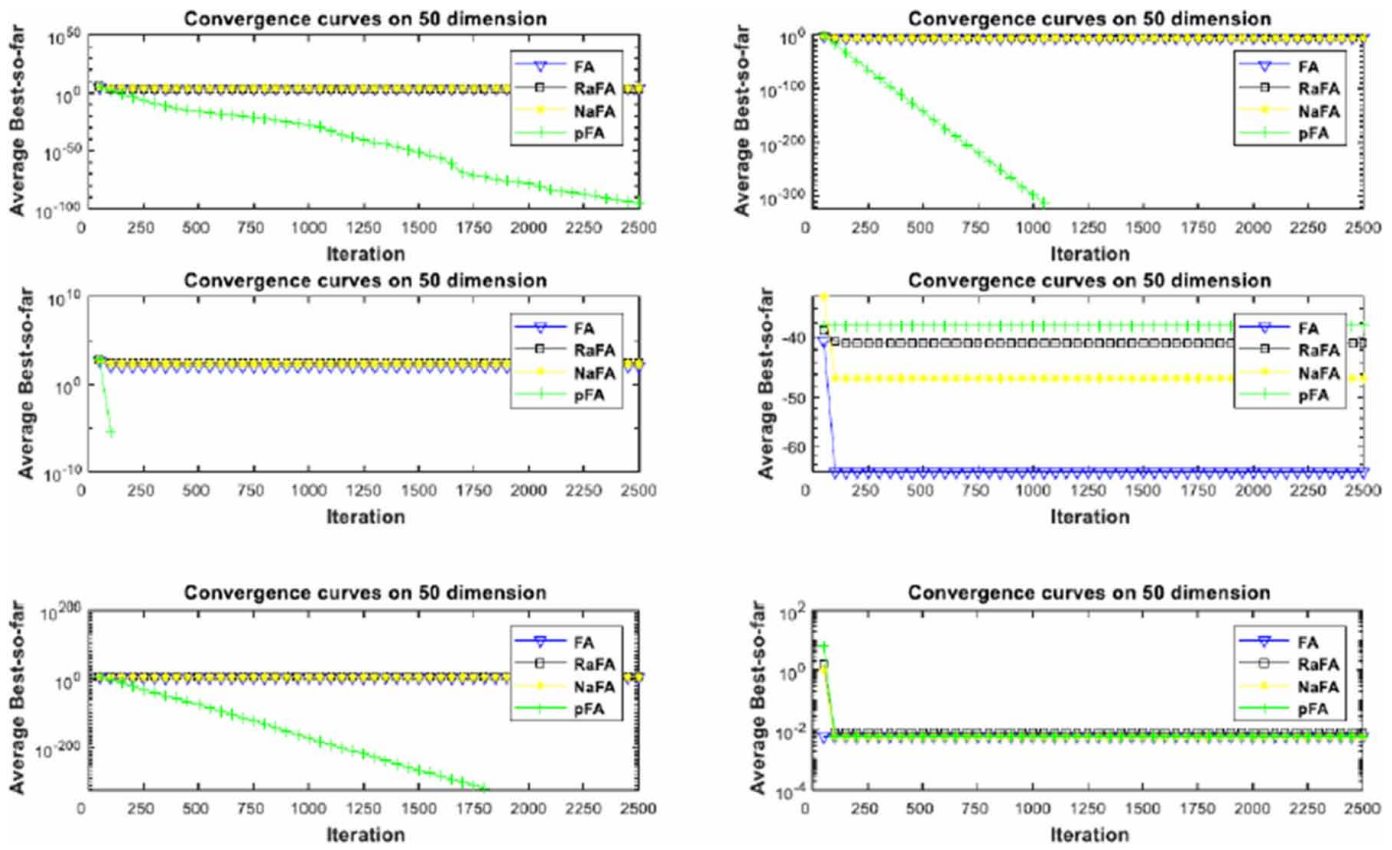
Figure 8. Convergence curve of $f_{3}, f_{7}, f_{11}, f_{15}, f_{19}$, and $f_{21}$ on 100 dimensions
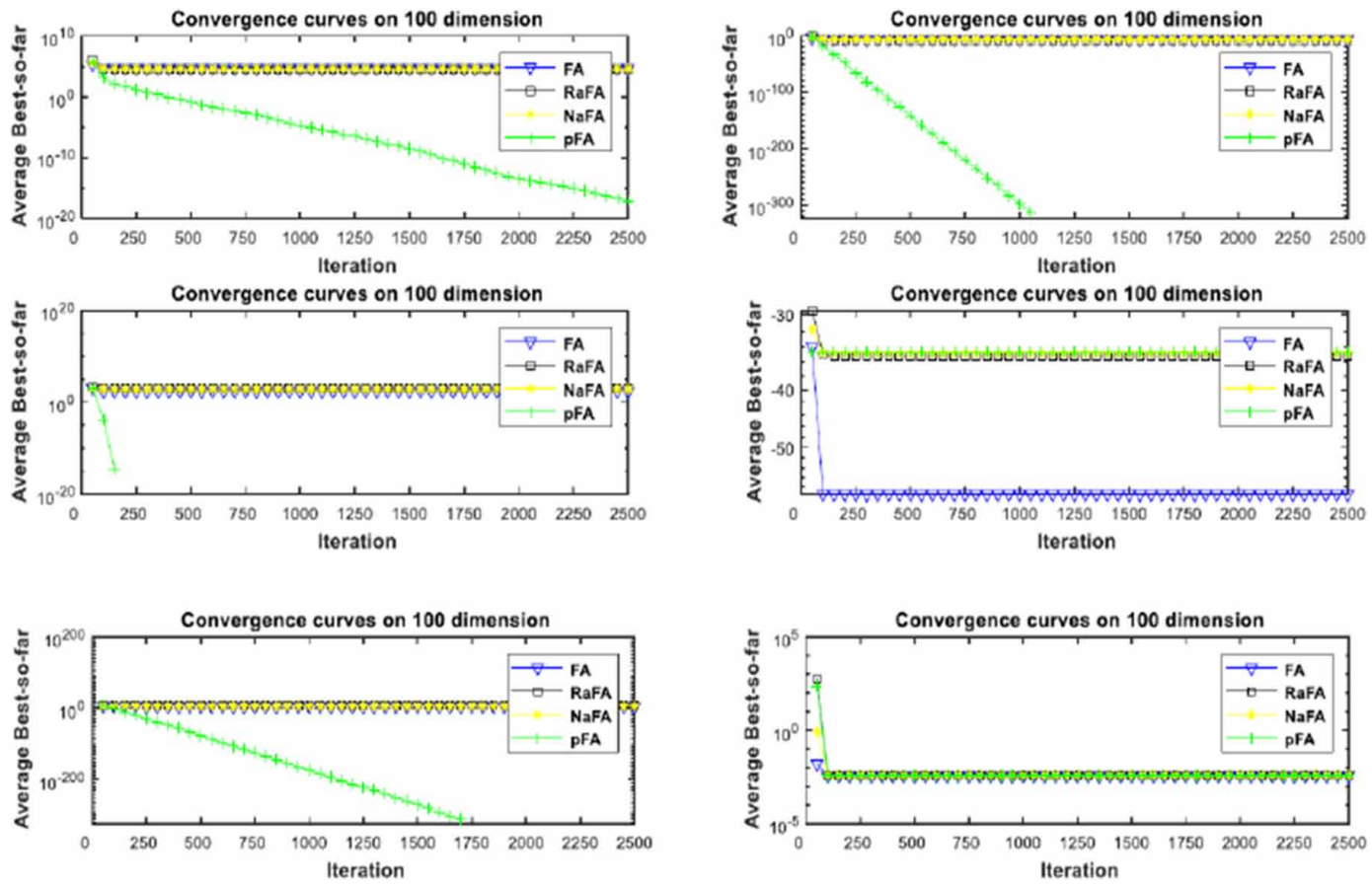

Table 7. Computational results of FA, RaFA, NaFA and pFAbased on probabilistic metric

\begin{tabular}{|c|c|c|c|c|}
\hline Function & Algorithm & $\mathbf{P}_{\text {better }}$ & $\mathbf{P}_{\text {equel }}$ & $\mathbf{P}_{\text {worse }}$ \\
\hline F3 & $\begin{array}{l}\text { FA } \\
\text { RaFA } \\
\text { NaFA } \\
\text { Pfa }\end{array}$ & $\begin{array}{l}1.00 \\
1.00 \\
1.00 \\
1.00\end{array}$ & $\begin{array}{l}0.00 \\
0.00 \\
0.00 \\
0.00\end{array}$ & $\begin{array}{l}0.00 \\
0.00 \\
0.00 \\
0.00\end{array}$ \\
\hline F7 & $\begin{array}{l}\text { FA } \\
\text { RaFA } \\
\text { NaFA } \\
\text { Pfa }\end{array}$ & $\begin{array}{l}1.00 \\
1.00 \\
1.00 \\
1.00\end{array}$ & $\begin{array}{l}0.00 \\
0.00 \\
0.00 \\
0.00\end{array}$ & $\begin{array}{l}0.00 \\
0.00 \\
0.00 \\
0.00\end{array}$ \\
\hline F11 & $\begin{array}{l}\text { FA } \\
\text { RaFA } \\
\mathrm{NaFA} \\
\mathrm{Pfa}\end{array}$ & $\begin{array}{l}1.00 \\
1.00 \\
1.00 \\
1.00\end{array}$ & $\begin{array}{l}0.00 \\
0.00 \\
0.00 \\
0.00\end{array}$ & $\begin{array}{l}0.00 \\
0.00 \\
0.00 \\
0.00\end{array}$ \\
\hline F15 & $\begin{array}{l}\text { FA } \\
\text { RaFA } \\
\text { NaFA } \\
\text { Pfa }\end{array}$ & $\begin{array}{l}0.00 \\
0.28 \\
0.01 \\
1.00\end{array}$ & $\begin{array}{l}0.00 \\
0.00 \\
0.00 \\
0.00\end{array}$ & $\begin{array}{l}1.00 \\
0.72 \\
0.99 \\
0.00\end{array}$ \\
\hline F19 & $\begin{array}{l}\text { FA } \\
\text { RaFA } \\
\text { NaFA } \\
\text { Pfa }\end{array}$ & $\begin{array}{l}1.00 \\
1.00 \\
1.00 \\
1.00\end{array}$ & $\begin{array}{l}0.00 \\
0.00 \\
0.00 \\
0.00\end{array}$ & $\begin{array}{l}0.00 \\
0.00 \\
0.00 \\
0.00\end{array}$ \\
\hline F21 & $\begin{array}{l}\text { FA } \\
\text { RaFA } \\
\text { NaFA } \\
\text { Pfa }\end{array}$ & $\begin{array}{l}1.00 \\
1.00 \\
1.00 \\
1.00\end{array}$ & $\begin{array}{l}0.00 \\
0.00 \\
0.00 \\
0.00\end{array}$ & $\begin{array}{l}0.00 \\
0.00 \\
0.00 \\
0.00\end{array}$ \\
\hline
\end{tabular}


Table 8. Computational results of DE and DEs

\begin{tabular}{|c|c|c|c|}
\hline Function & Algorithm & Mean & Std \\
\hline F1 & $\begin{array}{l}\text { DE } \\
\text { chDE } \\
\text { jDE } \\
\text { aDE } \\
\text { IMMSADE } \\
\text { pFA }\end{array}$ & $\begin{array}{l}6.54 \mathrm{e}-37 \\
1.02 \mathrm{e}-56 \\
3.24 \mathrm{e}-39 \\
2.63 \mathrm{e}-56 \\
5.68 \mathrm{e}-165 \\
0\end{array}$ & $\begin{array}{l}9.42 \mathrm{e}-37 \\
2.80 \mathrm{e}-56 \\
3.48 \mathrm{e}-39 \\
2.23 \mathrm{e}-56 \\
0 \\
0\end{array}$ \\
\hline F2 & $\begin{array}{l}\text { DE } \\
\text { chDE } \\
\text { jDE } \\
\text { aDE } \\
\text { IMMSADE } \\
\text { pFA }\end{array}$ & $\begin{array}{l}1.27 \mathrm{e}-05 \\
1.65 \mathrm{e}-04 \\
2.5800 \\
1.23 \mathrm{e}-01 \\
1.71 \mathrm{e}-137 \\
4.86 \mathrm{e}-55 \\
\end{array}$ & $\begin{array}{l}1.41 \mathrm{e}-05 \\
1.90 \mathrm{e}-04 \\
1.2800 \\
7.11 \mathrm{e}-02 \\
9.20 \mathrm{e}-137 \\
2.66 \mathrm{e}-54 \\
\end{array}$ \\
\hline F3 & $\begin{array}{l}\text { DE } \\
\text { chDE } \\
\text { jDE } \\
\text { aDE } \\
\text { IMMSADE } \\
\text { pFA }\end{array}$ & $\begin{array}{l}1.08 \text { e-33 } \\
7.16 \text { e-53 } \\
2.11 \text { e-36 } \\
2.93 \text { e-53 } \\
5.44 \text { e-161 } \\
0\end{array}$ & $\begin{array}{l}1.55 e-33 \\
2.08 \mathrm{e}-52 \\
2.58 \mathrm{e}-36 \\
2.68 \mathrm{e}-53 \\
2.93 \mathrm{e}-160 \\
0\end{array}$ \\
\hline F4 & $\begin{array}{l}\text { DE } \\
\text { chDE } \\
\text { jDE } \\
\text { aDE } \\
\text { IMMSADE } \\
\text { pFA }\end{array}$ & $\begin{array}{l}4.16 \text { e- } 18 \\
3.06 \text { e-34 } \\
3.40 \text { e- } 23 \\
6.75 \text { e-33 } \\
4.64 \text { e- } 48 \\
1.10 \text { e-321 }\end{array}$ & $\begin{array}{l}3.83 \mathrm{e}-18 \\
4.40 \mathrm{e}-34 \\
2.49 \mathrm{e}-23 \\
4.42 \mathrm{e}-33 \\
2.50 \mathrm{e}-47 \\
0 \\
\end{array}$ \\
\hline F5 & $\begin{array}{l}\text { DE } \\
\text { chDE } \\
\text { jDE } \\
\text { aDE } \\
\text { IMMSADE } \\
\text { pFA }\end{array}$ & $\begin{array}{l}1.99 \mathrm{e}-01 \\
1.02 \mathrm{e}+01 \\
1.91 \mathrm{e}-05 \\
2.77 \mathrm{e}-04 \\
3.14 \mathrm{e}-88 \\
4.82 \mathrm{e}-283\end{array}$ & $\begin{array}{l}3.39 \mathrm{e}-01 \\
5.0600 \\
1.12 \mathrm{e}-05 \\
1.10 \mathrm{e}-03 \\
1.69 \mathrm{e}-87 \\
0\end{array}$ \\
\hline F6 & $\begin{array}{l}\text { DE } \\
\text { chDE } \\
\text { jDE } \\
\text { aDE } \\
\text { IMMSADE } \\
\text { pFA }\end{array}$ & $\begin{array}{l}8.13 \mathrm{e}-31 \\
9.73 \mathrm{e}-51 \\
1.57 \mathrm{e}-33 \\
1.71 \mathrm{e}-50 \\
3.99 \mathrm{e}-165 \\
0\end{array}$ & $\begin{array}{l}8.51 \mathrm{e}-31 \\
1.97 \mathrm{e}-50 \\
1.69 \mathrm{e}-33 \\
1.74 \mathrm{e}-50 \\
0 \\
0\end{array}$ \\
\hline F7 & $\begin{array}{l}\text { DE } \\
\text { chDE } \\
\text { jDE } \\
\text { aDE } \\
\text { IMMSADE } \\
\text { pFA }\end{array}$ & $\begin{array}{l}1.49 \mathrm{e}-36 \\
7.19 \mathrm{e}-56 \\
5.34 \mathrm{e}-39 \\
5.15 \mathrm{e}-56 \\
9.09 \mathrm{e}-125 \\
0\end{array}$ & $\begin{array}{l}1.93 \mathrm{e}-36 \\
3.33 \mathrm{e}-55 \\
4.91 \mathrm{e}-39 \\
5.00 \mathrm{e}-56 \\
4.89 \mathrm{e}-124 \\
0\end{array}$ \\
\hline F8 & $\begin{array}{l}\text { DE } \\
\text { chDE } \\
\text { jDE } \\
\text { aDE } \\
\text { IMMSADE } \\
\text { pFA }\end{array}$ & $\begin{array}{l}0 \\
0 \\
0 \\
0 \\
0 \\
0\end{array}$ & $\begin{array}{l}0 \\
0 \\
0 \\
0 \\
0 \\
0\end{array}$ \\
\hline
\end{tabular}




\begin{tabular}{|l|l|l|l|}
\hline \multicolumn{1}{|c|}{ Function } & Algorithm & \multicolumn{1}{c|}{ Mean } & \multicolumn{1}{c|}{ Std } \\
\hline \multirow{5}{*}{ F9 } & DE & $4.32 \mathrm{e}-03$ & $1.30 \mathrm{e}-03$ \\
& chDE & $3.87 \mathrm{e}-03$ & $1.02 \mathrm{e}-03$ \\
& jDE & $4.88 \mathrm{e}-03$ & $1.42 \mathrm{e}-03$ \\
& aDE & $4.85 \mathrm{e}-03$ & $1.41 \mathrm{e}-03$ \\
& IMMSADE & $1.33 \mathrm{e}-04$ & $7.24 \mathrm{e}-05$ \\
& pFA & $4.32 \mathrm{e}-06$ & $4.04 \mathrm{e}-06$ \\
\hline \multirow{5}{*}{ F11 } & DE & 0 & 0 \\
& chDE & $2.46 \mathrm{e}-04$ & $1.33 \mathrm{e}-03$ \\
& jDE & 0 & 0 \\
\hline \multirow{5}{*}{ F13 } & aDE & 0 & 0 \\
& IMMSADE & 0 & 0 \\
& pFA & 0.0776 & 0.2426 \\
\hline & DE & $1.44 \mathrm{e}+02$ & $2.20 \mathrm{e}+01$ \\
& chDE & 2.5200 & 2.3100 \\
& jDE & 4.5900 & 3.5800 \\
& aDE & 0 & 0 \\
& IMMSADE & 0 & 0 \\
& pFA & 0 & 0 \\
\hline \multirow{5}{*}{ F21 } & DE & $1.35 \mathrm{e}-19$ & $2.40 \mathrm{e}-35$ \\
& chDE & $1.35 \mathrm{e}-19$ & $3.13 \mathrm{e}-29$ \\
& jDE & $1.35 \mathrm{e}-19$ & $2.41 \mathrm{e}-35$ \\
& aDE & $1.35 \mathrm{e}-19$ & $2.41 \mathrm{e}-35$ \\
& IMMSADE & $1.35 \mathrm{e}-19$ & $2.95 \mathrm{e}-32$ \\
& pFA & 0.5411 & 0.2116 \\
\hline & DE & $3.41 \mathrm{e}-01$ & $4.50 \mathrm{e}-02$ \\
& chDE & $1.60 \mathrm{e}-01$ & $4.55 \mathrm{e}-02$ \\
& jDE & $3.09 \mathrm{e}-01$ & $4.01 \mathrm{e}-02$ \\
& aDE & $3.18 \mathrm{e}-01$ & $4.11 \mathrm{e}-02$ \\
& IMMSADE & $6.10 \mathrm{e}-02$ & $1.72 \mathrm{e}-02$ \\
& pFA & $0.49 \mathrm{e}-01$ & $0.98 \mathrm{e}-01$ \\
\hline
\end{tabular}

\section{Key Point Localization}

Accurately locating the feature key points. After successful identification of key points in first stage, these points are further refined for accuracy by generating a different window sizes on basis of Taylor series and after doing this, if obtained extreme location intensity is greater than the user defined threshold (contrast threshold) then consider it or else discard. Similarly, any unwanted edges found after difference of Gaussian function, those locations are removed by $2 \times 2$ Hessian matrix as like in Harris corner detector. So only utmost edges and key points are obtained after set A and step B.

\section{Orientation Assignment}

Assigning orientation to the key points. In this step, orientation is traced between the key points based on their scale, gradient magnitude and direction. In this paper, around 36 bins are used to cover 360 degrees. From histogram of an image the highest intensity ' $\mathrm{H}$ ' is calculated and if any Key point intensity is above $80 \%$ of $\mathrm{H}$, then it will be considered in tracing.

\section{Key Point Descriptor}

Describing the key point as a high dimensional vector. Key point descriptor is obtained by local gradient which is generated in above three steps. The obtained gradient information is rotated to line up with 
the orientation of the key point and then weighted by Gaussian with variance of 1.5 key point scale. With this data, a collection of histograms is generated which are at center of key point. A total of 16 histograms which are arranged in the form of $4 \times 4$ grid and each grid carries 8 orientation bins. This leads to a feature vector of size 128. These vectors are called SIFT keys and used for identification of nearest possible object in an image. If more than three keys support a specific feature, that will be considered for further processes.

\section{TYPE-2 FUZZY SET}

The theory called as fuzzy set created by Zadeh. Fuzzy set theory prominently solves the problems related to uncertainty. However, because type-1 conventional membership functions of fuzzy set are determined, the uncertainty effects minimization is difficult by using any kind of algorithm and membership function. In pragmatic applications, usually the membership function is based on intuition empirically with a high degree of subjectivity. A generalized fuzzy set proposed by Zadeh to deal with the problems mentioned above i.e., type 2 fuzzy set. In a second type Fuzzy set the relation between membership function and element is fuzzy and uncertain. Therefore, a type-2 fuzzy set can describe the fuzzy membership function of uncertainty and the elements of uncertainty. Type- 2 fuzzy set can be defined as:

$\mathrm{B}=\left\{\left((\mathrm{y}, \mathrm{u}), \mu_{\mathrm{B}}(\mathrm{y}, \mathrm{u})\right) \mid \forall \mathrm{y} \in \mathrm{Y}, \forall \mathrm{u} \in \mathrm{Ky} \subseteq[0,1]\right\}$

where $\mathrm{B}$ denotes the type- 2 fuzzy set, $\mu \mathrm{B}(\mathrm{y}, \mathrm{u})$ denotes its type type- 2 membership function with $0 \leq$ $\mu_{\mathrm{B}}(\mathrm{y}, \mathrm{u}) \leq 1$. Ky denotes a primary membership function $\mu_{\mathrm{B}}\left(\mathrm{y}^{\prime}, \mathrm{u}\right)$ denotes the secondary membership function when $y=y^{\prime}$. However, the complexity of type- 2 fuzzy set is greater than that of type-1, in which more calculations are required. The problem exists while choosing secondary membership functions. In practical applications, a type-2 simplified fuzzy set often used, such as the interval type-2 fuzzy set (Mendel \& John, 2002). The membership functions of the element in type-2 fuzzy set is defined as:

$\mathrm{B}=\{((\mathrm{y}, \mathrm{u}), 1) \mid \forall \mathrm{y} \in \mathrm{Y}, \forall \mathrm{u} \in \mathrm{Ky} \subseteq[0,1]\}$

An alternative type-2 fuzzy set is defined as (with the help of membership functions of type-1):

$\mathrm{B}=\left(\mathrm{y}, \mu_{\mathrm{M}}(\mathrm{y}), \mu_{\mathrm{v}}(\mathrm{y})\right)\left|\forall \mathrm{y} \in \mathrm{Y} \mu_{\mathrm{M}}(\mathrm{y}) \leq \mu(\mathrm{y}) \leq \mu_{\mathrm{v}}(\mathrm{y})\right| \mathrm{u} \in[0,1]$

where $\mu(y)$ denotes an initial membership function of thype-1, B is the interval type-2 fuzzy set, and $\mu \mathrm{M}(\mathrm{y})$ and $\mu \mathrm{V}(\mathrm{y})$ is the upper and lower membership functions with respect to upper and lower envelopes of the interval membership function (type-2) and fuzzy set type-1 membership function can be obtained. Practically a pair to characterize reciprocal parameters i.e.:

$\mu_{\mathrm{M}}(\mathrm{y})=[\mu(\mathrm{y})] \alpha, \mu_{\mathrm{U}}(\mathrm{y})=[\mu(\mathrm{y})](1 / \alpha)$

where $\alpha$ is a fuzzy linguistic hedge $(\alpha \geq 1)$. Good output can be obtained when $\alpha \in[1,2]$. Footprint of uncertainty (FOU) can be represented by relating both type- 1 fuzzy set and type- 2 fuzzy set (Yang et al., 2016). The shape of type-2 fuzzy set is used in literature is represented by term FOU, therefore the distribution on the top of the shaded area is given by FOU. 


\section{FUSION RULE}

Let's assume two images $\mathrm{M}$ and $\mathrm{R}$ which is to be fused. After successful segmentation of both the images its corresponding segmented regions are represented as $M:\left\{S_{p, q}^{M}\right\}$ and $\mathrm{R}:\left\{S_{p, q}^{R}\right\}$ where $\mathrm{p}$ and q represents the segmented regions and its corresponding pixel positions respectively. The fusion rules play a significant role in performance of the proposed segmentation method. The earlier proposed image fusion rules are sensitivity to noise at high frequency i.e. edges, region boundaries and textures (Li \& Li, 2008) so in this paper, type 2 fuzzy logic is proposed which takes local and global information of the images. The shape of the membership function $\mu()$ is given as:

$$
\mu_{p, q}^{I}(i, j)=\frac{1}{1+\left|\frac{s_{p, q}^{I}(i, j)-c}{a}\right|^{2}}
$$

where $I=(M, R)$ and $(i, j)$ shows the spatial location of segmented images. Here, $\mathrm{c}$ is average of $\left(s_{p, q}^{I}\right)$ and 'a' is minimum of $\left(s_{p, q}^{I}\right)$. Next, at each position of the membership function, lower and upper membership functions $\mu_{L}(x)$ and $\mu_{U}(x)$ are calculated as follows:

$$
\left\{\begin{array}{l}
\mu_{L}^{I, p, q}(x, y)=\left[\mu_{p, q}^{I}(i, j)\right]^{\alpha} \\
\mu_{U}^{I, p, q}(x, y)=\left[\mu_{p, q}^{I}(i, j)\right]^{1 / \alpha}
\end{array}\right.
$$

Fuzzy set performance is measured on the basis of fuzzy entropy, a high value of fuzzy entropy shows the best fuzzy set and low value of fuzzy entropy shows wicked fuzzy set (Bhateja et al;, 2015). Hence a higher value of fuzzy entropy leads to better image fusion rules. The detailed description about the fuzzy entropy already discussed in section 2 .

\section{RESULTS AND DISCUSSIONS}

The performance of proposed method is evaluated by performing experiments on five pairs of images related to different diseases of brain. In Set-A, CT and T2- Weighted MR images of brain tumor related to neoplastic disease. In Set-B, CT, T2-Weighted MR images of brain stroke due to cerebrovascular disease. In Set-C, depicts the T1-Weighted MR and T2-Weighted MR images of brain related to Alzheimer disease. In Set-D, depicts the T1-Weighted MR and T2-Weighted MR images of brain related to fatal disease, while in Set-E, represents the CT, T1-Weighted MR brain images due to sarcoma disease. The medical images used in the research were obtained from https:// www.imagefusion.org/ and http://www.med.harvard.edu/aanlib/home.html. All the simulations are performed on HP Compaq LE1902X personal computer with Matlab version 2016a.

\section{Measuring Parameters: Standard Deviation (STD)}

Standard deviation is the estimation of gray contrast in the combined image. At the point when the estimated $\mathrm{S}$ is high, the fused image will have high contrast. When $\mathrm{p}(\mathrm{k})$ is the probability of gray level $\mathrm{k}$ and $\mathrm{k}_{\mathrm{m}}$ is the mean of $\mathrm{k}[33]$ : 
$\mathrm{STD}=\sqrt{\sum_{K=0}^{J-1}\left(k-k_{m}\right)^{2} p(k)}$

\section{Structural Similarity Index (SSIM)}

Structural similarity index is used for the estimation of similarity or closeness between two images. It is used to measure quality by comparing one image provided with the other image. It is regarded as an excellent quality. It is the upgraded version of universal image quality index. Consider D1 = $\left(\mathrm{m}_{1} \mathrm{~N}\right)^{2}$ and $\mathrm{D} 2=\left(\mathrm{m}_{2} \mathrm{~N}\right)^{2}$ are the two variables to make the division stable by making the denominator weak. $\mathrm{N}$ represents the dynamic range values of pixels. The general values for $\mathrm{m}_{1}$ and $\mathrm{m}_{2}$ are 0.01 and 0.03 respectively. If ' $f$ ' is the fused image and $r$ is the reference image:

$$
S S I M=\frac{(2 r 2 f+D 1)(2 r 2 f+D 2)}{(2 r 2 f+D 1)(2 r+2 f+D 2)}
$$

\section{Mutual Information (MI)}

Mutual information is utilized as a measure to know the performance in multimodal fusion (Bhateja et al., 2015). If the value of MI is high, then it suggests that the combined frame constraints more parental image information. $\mathrm{p}(\mathrm{m}), \mathrm{p}(\mathrm{n})$ are the marginal probability distribution functions of both the images, $\mathrm{p}(\mathrm{m}, \mathrm{n})$ is the joint probability distribution function:

$$
M I(m, n)=\sum_{m \in M} \sum_{n \in N} p(m, n) \log \left(\frac{p(m, n)}{p(m) p(n)}\right)
$$

where $\mathrm{M}$ and $\mathrm{N}$ are number of rows and columns of both the images.

\section{Entropy}

It is defined as the amount of information which the image contains. It can be used to measure the quality of the output fused image. The entropy can be known if probability density (p) of pixel levels is known. If the entropy is higher, then fusion is efficient (Bhateja et al., 2015):

$$
\text { Entropy }=-\sum_{i=0}^{K-1} p(m i) \log p(m i)
$$

\section{Edge Based Similarity Measure ( $\left.Q^{A B / F}\right)$}

It can be used to measure the edge details in a fused image. The value of Q is close to 1 for a highquality fused image. A number of ways are introduced to get the edge information of the image like simple edge detection algorithm, local gradients and many more (Bhateja et al., 2015). It is calculated with below equation:

$$
Q^{A B / F}=\frac{\sum_{i=1}^{x} \sum_{j=1}^{y} Q a(i, j) W a(i, j)+Q(i, j) W b(i, j)}{\sum_{i=1}^{x} \sum_{j=1}^{y} W a(i, j)+W b(i, j)}
$$


where:

$\mathrm{Qa}(\mathrm{i}, \mathrm{j})=\mathrm{Qa}_{\mathrm{g}}(\mathrm{x}, \mathrm{y}) \mathrm{Qa} \mathrm{a}_{\alpha}(\mathrm{x}, \mathrm{y}), \mathrm{Qb}(\mathrm{i}, \mathrm{j})=\mathrm{Qb}(\mathrm{x}, \mathrm{y}) \mathrm{Qb}_{\alpha}(\mathrm{x}, \mathrm{y})$

\section{Correlation Coefficient (CC)}

This parameter is used to measure the closeness between reference image and fused image. If the reference image and fused image are identical then the value of correlation coefficient is nearly equal to 1 (Bhateja et al., 2015). If the difference between the fused image and reference image is additional, then the value of correlation coefficient will be less than 1 :

$$
C=\frac{\sum_{i=1}^{j}\left(m_{i}-M\right)}{\sqrt{\sum_{i=1}^{j}\left(m_{i}-M\right)^{2} \sum_{i=1}^{j}\left(n_{i}-N\right)^{2}}}
$$

where $\mathrm{M}$ and $\mathrm{N}$ are mean values of corresponding images.

\section{Experiments on CT/MRI Image Fusion}

\section{Neoplastic Disease}

Neoplastic diseases are conditions that cause tumor growth. It is the kind of tumor due to extreme development of cells in the brain. They can be benign and malignant. Benign tumors are non-cancerous and grow slowly and spread less to other organs. Malignant tumors are cancerous and can spread to other tissues or organs. If not detected in the early stage, it may be life threatening. Identifying such kind of growth at early stage, may not lead to cancer. If such kind of growth is malignant, it will spread to all other parts of brain at quick rate leads to cancer. From Figure 9, such kind of tumor

Figure 9. (a) Input images; (b) Segmented images: Fused images; (c) With PSO; (d) With QPSO; (e) With HBMO; (f) With pFA

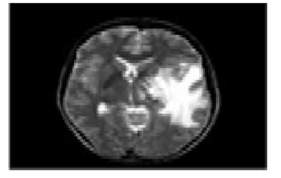

(a)

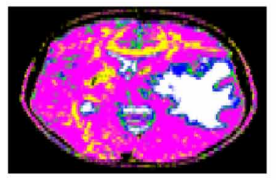

(c)
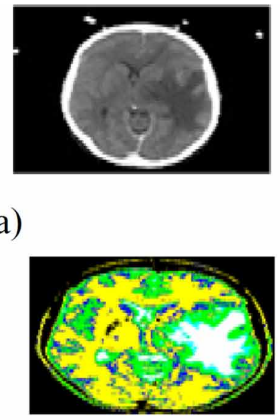

(d)

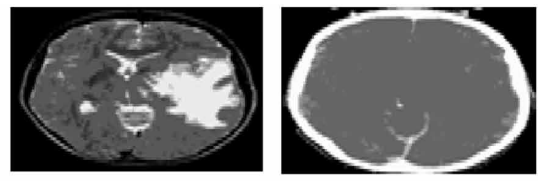

(b)

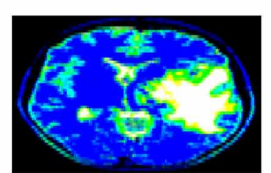

(e)

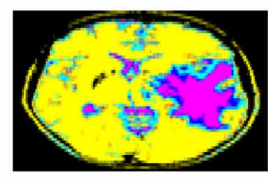

(f)

growth is identified better with the proposed algorithm as compared to others and Table 9 shows the performance of the proposed algorithm in FSIM, SSIM, Entropy, PSNR, $\mathrm{Q}^{\mathrm{AB} / \mathrm{F}}$ and MI.

\section{Cerebrovascular Disease}

Cerebrovascular disease refers to conditions that affect the flow of blood through the brain and can lead to stroke. Hypertension (high blood pressure) is the most important risk factor for stroke 
Table 9. Set A: Neoplastic disease

\begin{tabular}{|l|c|l|c|c|c|c|c|c|}
\hline Method & FSIM & ENTROPY & PSNR & SSIM & STD & \multicolumn{1}{|c|}{ Q $^{\text {AB/F }}$} & MI & CC \\
\hline PSO & 0.893427 & 21.064733 & 34.32786 & 0.91319 & 0.189056 & 0.620952 & 3.23917 & 0.62095 \\
\hline QPSO & 0.956501 & 21.109136 & 34.36472 & 0.914872 & 0.206056 & 0.663134 & 3.28259 & 0.66313 \\
\hline HBMO & 0.958995 & 21.134748 & 34.38426 & 0.934355 & 0.234453 & 0.683590 & 3.28660 & 0.68359 \\
\hline Proposed & 0.978974 & 21.325112 & 34.38888 & 0.947851 & 0.238101 & 0.696854 & 3.29874 & 0.68987 \\
\hline
\end{tabular}

and cerebrovascular disease. This disease is permanently or temporarily effected by some of the reasons like ischemia, area of brain, blood bleeding and in pathological process more than two blood vessels involvement. It includes aneurysms, vertebral stenosis, carotid stenosis and, stroke, vascular malformations and intracranial stenosis. These diseases can be prevented by medication and lifestyle changes. Blood thinners and other modalities, including surgery are used to treat strokes. Figure 10 shows the effectiveness of proposed algorithm in visual quality and Table 10 shows in all measured parameters proposed is better.

Figure 10. (a) Input images; (b) Segmented images: Fused images; (c) With PSO; (d) With QPSO; (e) With HBMO; (f) With pFA

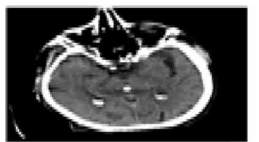

(a)

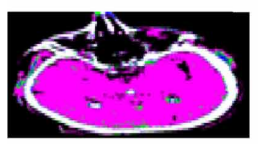

(c)
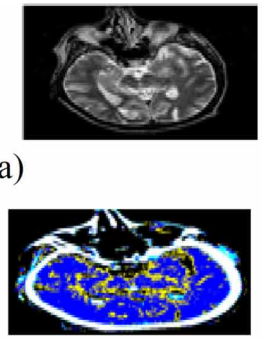

(d)

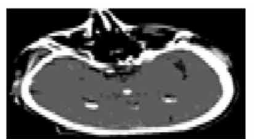

(b)

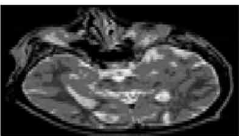

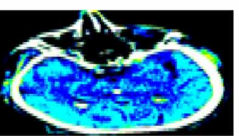

(e)

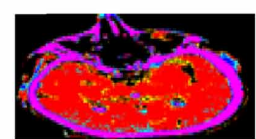

(f)

Table 10. Set B: Cerebrovascular disease

\begin{tabular}{|l|c|l|c|c|c|c|c|c|}
\hline Method & FSIM & ENTROPY & PSNR & SSIM & STD & Q $^{\mathbf{A B} / \mathbf{F}}$ & MI & CC \\
\hline PSO & 0.991425 & 22.640391 & 34.39331 & 0.94834 & 0.137244 & 0.594891 & 3.30639 & 0.59489 \\
\hline QPSO & 0.960303 & 22.679535 & 34.58154 & 0.949456 & 0.152098 & 0.604217 & 3.34325 & 0.60422 \\
\hline HBMO & 0.942479 & 22.689466 & 34.58518 & 0.953553 & 0.183669 & 0.683029 & 3.34592 & 0.68303 \\
\hline Proposed & 0.975484 & 22.754874 & 34.60245 & 0.965874 & 0.112457 & 0.685874 & 3.35015 & 0.69999 \\
\hline
\end{tabular}

\section{Alzheimer Disease}

Alzheimer's disease is an irreversible progressive brain disorder that slowly destroys memory and thinking skills eventually, the ability to carry out even simple tasks. It usually appears first in mid 60s. There is no cure for this disease, but treatment can help slow the progression of the disease and improve quality of life. In general, this disease is diagnosed by testing metal stability manually or eye tracing test or by MRI scan or by CT scan. A small structural difference in brain leads to disease, so identifying such kind of small difference is a big task for the researchers. In this paper, MRI and 
CT scan images are fused for better identification of small differences in brain which leads to early diagnosis of Alzheimer.T1-Weighted MR image of 70 years old man and T2-Weighted MR images of 73 years old man is considered for performance test of proposed method. The qualitative result of the proposed algorithm is well explained in the Figure 11. From the Figure 11, the visual quality of Alzheimer disease is better with proposed as compared with other algorithms. The quantitative results of the proposed is as shown in Table 11. It shows the superiority in all aspects.

Figure 11. (a) Input images; (b) Segmented images: Fused images; (c) With PSO; (d) With QPSO; (e) With HBMO; (f) With pFA

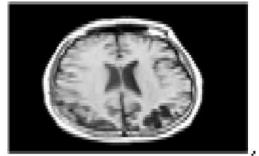

(a)

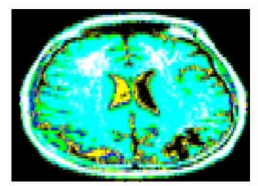

(c)
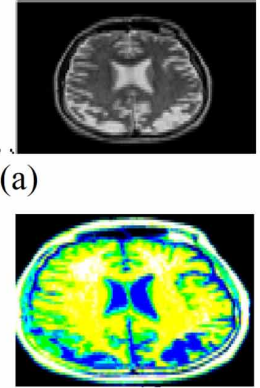

(d)

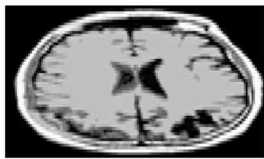

(b)

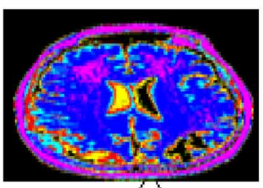

(e)
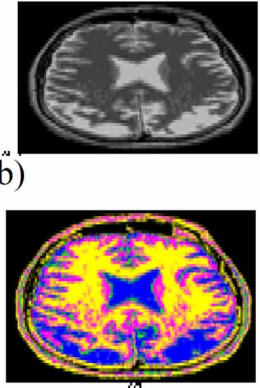

(f)

Table 11. Set C: Alzheimer disease

\begin{tabular}{|l|c|l|c|c|c|c|c|c|}
\hline Method & FSIM & ENTROPY & PSNR & SSIM & STD & \multicolumn{1}{|c|}{ Q $^{\mathbf{A B} / \mathbf{F}}$} & MI & CC \\
\hline PSO & 0.336717 & 21.390594 & 32.82359 & 0.911531 & 0.777079 & 0.719411 & 3.1012 & 0.71941 \\
\hline QPSO & 0.444859 & 21.405167 & 32.83409 & 0.937020 & 0.444859 & 0.720572 & 3.1255 & 0.72057 \\
\hline HBMO & 0.660098 & 21.485016 & 32.85253 & 0.951837 & 0.660980 & 0.725686 & 3.1454 & 0.72569 \\
\hline Proposed & 0.612444 & 21.489999 & 32.87854 & 0.959658 & 0.325697 & 0.747844 & 3.1665 & 0.73687 \\
\hline
\end{tabular}

\section{Fatal Disease}

A fatal disease has no cure and ultimately results in death of the patient. Death can occur within a few hours to several years, depending upon the particular disease. Some examples of fatal diseases include cancer, severe heart disease, AIDS, dementia etc. As there is no cure, the patients can only be given supportive and palliative treatment. In this paper Pick's disease is consider as a fatal disease. Pick's disease is a rare disease which leads to loss of memory, reduction in thinking levels, remembering the language and abnormal behavior of human beings. The reason is some minor damage in brain tissues. This can be identified by fusing CT and MRI images and was successfully done with the proposed method and compared the results with other soft-computing techniques. Form Table 12 and Figure 12 it is observed the proposed method effectiveness in fusing the images.

\section{Sarcoma Disease}

Sarcoma is a malignant tumor, a rare kind of cancer. Sarcomas grow in connective tissue cells, for example fat, blood vessels, nerves, bones, muscles and cartilage. They are treated by surgery to remove the tumor and radiotherapy and chemotherapy. Sarcomas are usually incurable and can be deadly. From Figure 13, visual fused image quality obtained with pFA is far better than fused images obtained with PSO, QPSO and HBMO. The green color regions in Figure 13(f) shows the tumor 
Figure 12. (a) Input images; (b) Segmented images: Fused images; (c) With PSO; (d) With QPSO; (e) With HBMO; (f) With pFA
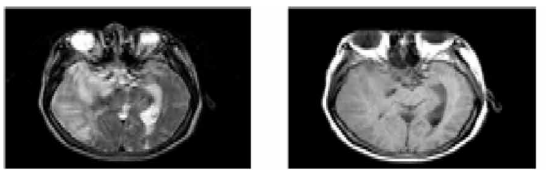

(a)

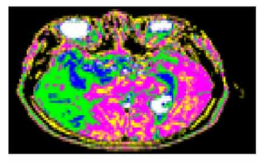

(c)

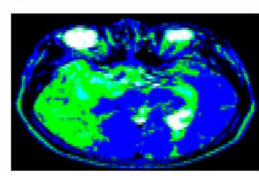

(d)
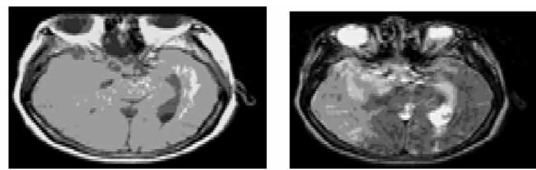

(b)

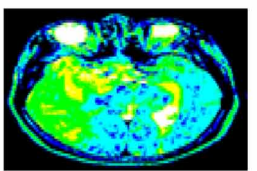

(e)

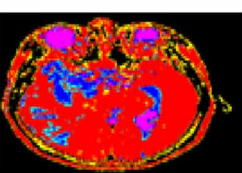

(f)

Table 12. Set D: Fatal disease

\begin{tabular}{|l|c|l|c|c|c|c|c|c|}
\hline Method & \multicolumn{1}{|c|}{ FSIM } & ENTROPY & PSNR & SSIM & STD & Q $^{\text {AB/F }}$ & MI & CC \\
\hline PSO & 0.307900 & 22.177216 & 32.56973 & 0.897339 & 0.437479 & 0.742869 & 3.54065 & 0.74287 \\
\hline QPSO & 0.437479 & 22.254970 & 32.63030 & 0.920576 & 0.833801 & 0.756198 & 3.54868 & 0.75620 \\
\hline HBMO & 0.693950 & 22.278068 & 32.78759 & 0.924099 & 0.307951 & 0.768028 & 3.59584 & 0.76803 \\
\hline Proposed & 0.712457 & 22.358744 & 32.89765 & 0.932547 & 0.547844 & 0.77487 & 3.60124 & 0.78975 \\
\hline
\end{tabular}

Figure 13. (a) Input images; (b) Segmented images: Fused images; (c) With PSO; (d) With QPSO; (e) With HBMO; (f) With pFA
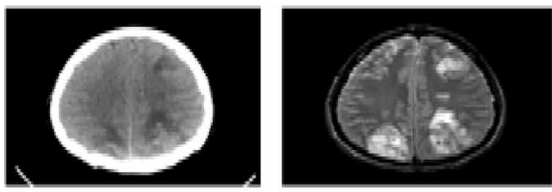

(a)

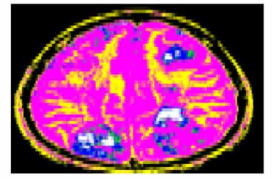

(c)

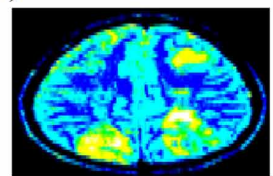

(d)
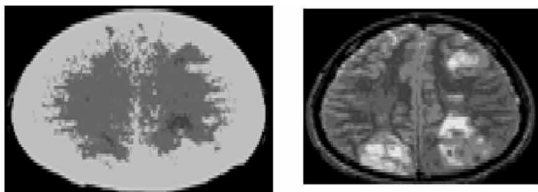

(b)

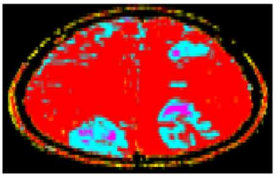

(e)

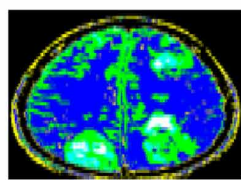

(f)

effected regions which is top highlighted with proposed pFA. The proposed method is better not only in visual quality but also in other fusion measuring parameters as shown in Table 13.

From the above discussions, results and experiments, it is found that proposed pFA gives advanced amount of mutual information (MI) by image thresholding and Scale-Invariant Feature Transform. The experimental results show that the proposed fusion technique has significant quantitative improvement than that of the existing fusion techniques. This fusion algorithm is tested for medical imaging applications and it is also well suited for other applications like remote sensing and development of region of area. The proposed technique can be used for satellite imaging applications such as weather forecasting, forest degradation and object tracking applications. The proposed algorithm overcomes the limitations of PSO, QPSO and HBMO. 
Table 13. Set E: Sarcoma disease

\begin{tabular}{|l|l|l|l|l|l|l|l|l|}
\hline \multicolumn{1}{|c|}{ Method } & \multicolumn{1}{|c|}{ FSIM } & \multicolumn{1}{|c|}{ ENTROPY } & \multicolumn{1}{|c|}{ PSNR } & \multicolumn{1}{|c|}{ SSIM } & \multicolumn{1}{|c|}{ STD } & \multicolumn{1}{c|}{$\mathbf{Q}^{\mathrm{AB} / \mathrm{F}}$} & MI & CC \\
\hline PSO & 0.21906 & 21.23275 & 33.691 & 0.89593 & 0.24321 & 0.649 & 2.8722 & 0.6490 \\
\hline QPSO & 0.24321 & 21.25676 & 33.708 & 0.90873 & 0.32883 & 0.6583 & 2.8922 & 0.6583 \\
\hline HBMO & 0.32883 & 21.39504 & 33.767 & 0.92194 & 0.21906 & 0.6615 & 2.8956 & 0.6615 \\
\hline Proposed & 0.396587 & 21.45878 & 33.801 & 0.92878 & 0.26587 & 0.6745 & 2.90124 & 0.6754 \\
\hline
\end{tabular}

\section{CONCLUSION}

In this paper, two MRI images are fused for better diagnosis of diseases like neoplastic disease, cerebrovascular disease, Alzheimer disease, fatal disease, sarcoma disease. The two images are segmented on the basis of 2-D histogram with pFA by assuming Renyi entropy as objective function. The pFA is used to get optimal thresholds for better segmentation. The segmented regions of both the images are extracted with the Scale Invariant Feature Transform (SIFT) algorithm and on the basis of interval type- 2 fuzzy set fusion rules both the images are fused. From the experiments, proposed algorithm is better in visual quality for diagnosing diseases and better in mutual information, Edge based similarity measure and correlation coefficient as compared to other algorithms. 


\section{REFERENCES}

Al-Tayyan, A., Assaleh, K., \& Shanableh, T. (2017). Decision-level fusion for single-view gait recognition with various carrying and clothing conditions. Image and Vision Computing, 61, 54-69. doi:10.1016/j. imavis.2017.02.004

Bhateja, V., Patel, H., Krishn, A., Sahu, A., \& Lay-Ekuakille, A. (2015). Multimodal medical image sensor fusion framework using cascade of wavelet and contourlet transform domains. IEEE Sensors Journal, 15(12), 6783-6790. doi:10.1109/JSEN.2015.2465935

Brest, J., Greiner, S., Boskovic, B., Mernik, M., \& Zumer, V. (2006). Self-adapting control parameters in differential evolution: A comparative study on numerical benchmark problems. IEEE Transactions on Evolutionary Computation, 10(6), 646-657. doi:10.1109/TEVC.2006.872133

Chiranjeevi, K., \& Jena, U. (2017). Hybrid gravitational search and pattern search-based image thresholding by optimising Shannon and fuzzy entropy for image compression. International Journal of Image and Data Fusion, 8(3), 236-269. doi:10.1080/19479832.2017.1338760

Chiranjeevi, K., \& Jena, U. (2018). SAR image compression using adaptive differential evolution and pattern search based K-means vector quantization. Image Analysis \& Stereology, 37(1), 35-54. doi:10.5566/ias.1611

Choi, M., Kim, R. Y., Nam, M. R., \& Kim, H. O. (2005). Fusion of multispectral and panchromatic satellite images using the curvelet transform. IEEE Geoscience and Remote Sensing Letters, 2(2), 136-140. doi:10.1109/ LGRS.2005.845313

El-Hoseny, H. M., El-Rahman, W. A., El-Rabaie, E. S. M., El-Samie, F. E. A., \& Faragallah, O. S. (2018). An efficient DT-CWT medical image fusion system based on modified central force optimization and histogram matching. Infrared Physics \& Technology, 94, 223-231. doi:10.1016/j.infrared.2018.09.003

Ghassemian, H. (2016). A review of remote sensing image fusion methods. Information Fusion, 32, 75-89. doi:10.1016/j.inffus.2016.03.003

Haddadpour, M., Daneshvar, S., \& Seyedarabi, H. (2017). PET and MRI image fusion based on combination of 2-D Hilbert transform and IHS method. Biomedical Journal, 40(4), 219-225. doi:10.1016/j.bj.2017.05.002 PMID:28918910

He, Y., Deng, G., Wang, Y., Wei, L., Yang, J., Li, X., \& Zhang, Y. (2018). Optimization of SIFT algorithm for fast-image feature extraction in line-scanning ophthalmoscope. Optik (Stuttgart), 152, 21-28. doi:10.1016/j. ijleo.2017.09.075

Jin, X., Jiang, Q., Yao, S., Zhou, D., Nie, R., Hai, J., \& He, K. (2017). A survey of infrared and visual image fusion methods. Infrared Physics \& Technology, 85, 478-501. doi:10.1016/j.infrared.2017.07.010

Karri, C., \& Jena, U. (2016). Fast vector quantization using a Bat algorithm for image compression. Engineering Science and Technology, an International Journal, 19(2), 769-781. doi:10.1016/j.jestch.2015.11.003

Lang, J., \& Hao, Z. (2014). Novel image fusion method based on adaptive pulse coupled neural network and discrete multi-parameter fractional random transform. Optics and Lasers in Engineering, 52, 91-98. doi:10.1016/j. optlaseng.2013.07.005

Li, L., \& Li, D. (2008). Fuzzy entropy image segmentation based on particle swarm optimization. Progress in Natural Science, 18(9), 1167-1171. doi:10.1016/j.pnsc.2008.03.020

Liang, Q., \& Mendel, J. M. (2000). Interval type-2 fuzzy logic systems: Theory and design. IEEE Transactions on Fuzzy Systems, 8(5), 535-550. doi:10.1109/91.873577

Lowe, D. G. (1999, September). Object recognition from local scale-invariant features. In ICCV (Vol. 99, No. 2, pp. 1150-1157). Academic Press. doi:.10.1109/ICCV.790410

Mendel, J. M., \& John, R. B. (2002). Type-2 fuzzy sets made simple. IEEE Transactions on Fuzzy Systems, 10(2), 117-127. doi:10.1109/91.995115

Naidu, V. P. S., \& Raol, J. R. (2008). Pixel-level image fusion using wavelets and principal component analysis. Defence Science Journal, 58(3), 338-352. doi:10.14429/dsj.58.1653 
Nirmala, D. E., \& Vaidehi, V. (2015, March). Comparison of Pixel-level and feature level image fusion methods. In Proceedings of the 2015 2nd international conference on computing for sustainable global development (INDIACom) (pp. 743-748). IEEE.

Noman, N., Bollegala, D., \& Iba, H. (2011, June). An adaptive differential evolution algorithm. In Proceedings of the 2011 IEEE Congress of Evolutionary Computation (CEC) (pp. 2229-2236). IEEE. doi:10.1109/ CEC.2011.5949891

Petrovic, V. S., \& Xydeas, C. S. (2004). Gradient-based multiresolution image fusion. IEEE Transactions on Image Processing, 13(2), 228-237. doi:10.1109/TIP.2004.823821 PMID:15376943

Rényi, A. (1961, June). On measures of information and entropy. In Proceedings of the 4th Berkeley symposium on mathematics, statistics and probability (Vol. 1). Academic Press.

Sarkar, S., \& Das, S. (2013). Multilevel image thresholding based on 2D histogram and maximum Tsallis entropy-A differential evolution approach. IEEE Transactions on Image Processing, 22(12), 4788-4797. doi:10.1109/TIP.2013.2277832 PMID:23955760

Wan, T., Zhu, C., \& Qin, Z. (2013). Multifocus image fusion based on robust principal component analysis. Pattern Recognition Letters, 34(9), 1001-1008. doi:10.1016/j.patrec.2013.03.003

Wang, H., Wang, W., Sun, H., \& Rahnamayan, S. (2016). Firefly algorithm with random attraction. International Journal of Bio-inspired Computation, 8(1), 33-41. doi:10.1504/IJBIC.2016.074630

Wang, H., Wang, W., Zhou, X., Sun, H., Zhao, J., Yu, X., \& Cui, Z. (2017). Firefly algorithm with neighborhood attraction. Information Sciences, 382, 374-387. doi:10.1016/j.ins.2016.12.024

Wang, S., Li, Y., \& Yang, H. (2017). Self-adaptive differential evolution algorithm with improved mutation mode. Applied Intelligence, 47(3), 644-658. doi:10.1007/s10489-017-0914-3

Xu, Z. (2014). Medical image fusion using multi-level local extrema. Information Fusion, 19, 38-48. doi:10.1016/j. inffus.2013.01.001

Yang, L., Guo, B. L., \& Ni, W. (2008). Multimodality medical image fusion based on multiscale geometric analysis of contourlet transform. Neurocomputing, 72(1-3), 203-211. doi:10.1016/j.neucom.2008.02.025

Yang, S., Wang, M., Lu, Y., Qi, W., \& Jiao, L. (2009). Fusion of multiparametric SAR images based on SWnonsubsampled contourlet and PCNN. Signal Processing, 89(12), 2596-2608. doi:10.1016/j.sigpro.2009.04.027

Yang, Y., Que, Y., Huang, S., \& Lin, P. (2016). Multimodal sensor medical image fusion based on type-2 fuzzy logic in NSCT domain. IEEE Sensors Journal, 16(10), 3735-3745. doi:10.1109/JSEN.2016.2533864

Yu, S., Zhu, S., Ma, Y., \& Mao, D. (2015). A variable step size firefly algorithm for numerical optimization. Applied Mathematics and Computation, 263, 214-220. doi:10.1016/j.amc.2015.04.065

Zadeh, L. A. (1965). Fuzzy sets. Information and Control, 8(3), 338-353. doi:10.1016/S0019-9958(65)90241-X

Zhenyu, G., Bo, C., Min, Y., \& Binggang, C. (2006, September). Self-adaptive chaos differential evolution. In Proceedings of the International Conference on Natural Computation (pp. 972-975). Springer. doi:10.1007/11881070_128 
M.V. Srikanth is a Research Scholar, pursuing a PhD in the field of Image Processing from Jawaharlal Nehru Technological University, Kakinada, Andhra Pradesh, India. He has been working as an Academician in the capacity of an Assistant Professor in Electronics and Communication department of Gudlavalleru Engineering College, a reputed Autonomous Engineering College in Andhra Pradesh, India. Apart from regular academia, with a zeal to explore new technologies and a penchant towards pursuing research in the field of image processing it has led him to do research work in a governmental organization in Andhra Pradesh. His interests are inclined more towards signal processing and embedded systems.

V.V.K.D.V. Prasad, Dr., is working as a Professor and Head of the Department of Electronics \& Communication Engineering, in Gudlavalleru Engineering College, an Autonomous NBA accredited College in Andhra Pradesh, India. He received Ph. D for his work in Signal Processing in 2011 from Jawaharlal Nehru technological University, Kakinada, India. His areas of interest include signal processing, electrostatics, electromagnetic fields, and transmission lines. He developed an abstract technical trait that addresses various other fields where digitalization can be achieved. His research findings are in the methodology used, problems encountered and the practical implications of composite features and filtering coefficients in advanced filters.

K. Satya Prasad received a B.Tech. degree in Electronics and Communication Engineering from JNTU college of Engineering, Anantapur, Andhra Pradesh, India in 1977 and M.E. degree in Communication Systems from Guindy college of Engg., Madras University, Chennai, India in 1979 and PhD from Indian Institute of Technology, Madras in 1989. He started his teaching career as a Teaching Assistant at the Regional Engineering College, Warangal in 1979. He joined JNT University, Hyderabad as a Lecturer in 1980 and served in different constituent colleges such as Kakinada, Hyderabad, and Anantapur and in different capacities such as Associate Professor, Professor, Head of the Department, Vice Principal, and Principal. At JNTUK University he served as the Director of Evaluation, Director IST and Rector. He has published more than 250 technical papers in various national and international conferences and journals, and authored four textbooks. He has guided 35 PhD scholars and at present 15 scholars are working with him. His areas of research include communications, signal processing, image processing, neural networks, ad-hoc wireless networks, etc. Dr Prasad was a recipient of the Andhra Pradesh State Teacher Award in 2009 and received different awards at a national level. Dr. Prasad is a Fellow member of various professional bodies like IEEE, IETE, IE (I), and ISTE. After retiring from JNTUK service, Prasad worked as a Pro Vice Chancellor of KL University. At present, he is working as a Rector of Vignan's Foundation for Science, Technology and Research, Vadlamudi, Guntur, AP. 